\title{
Redatuming controlled-source electromagnetic data using Stratton-Chu type integral transformations
}

\author{
Michael Zhdanov ${ }^{\mathrm{a}, \mathrm{b}, \mathrm{c}}$, Hongzhu Cai ${ }^{\mathrm{a}, *}$ \\ a Consortium for Electromagnetic Modeling and Inversion (CEMI), University of Utah, Salt Lake City, UT 84112, USA \\ b TechnoImaging, Salt Lake City, UT 84107, USA \\ c Moscow Institute of Physics and Technology, Moscow 141700, Russia
}

\section{A R T I C L E I N F O}

\section{Article history:}

Received 27 June 2015

Received in revised form 2 December 2015

Accepted 5 January 2016

Available online 9 January 2016

\section{Keywords:}

Electromagnetic redatuming

Analytical continuation

Interpolation

Electromagnetic theory

\begin{abstract}
A B S T R A C T
We present a new method of analyzing controlled-source electromagnetic (CSEM) data based on redatuming of the observed data from the actual receivers into the virtual receivers. We use the Stratton-Chu type integral transform to calculate the EM field in the virtual receivers. The virtual receivers can be placed at any desirable position, including close to the target, which increases the sensitivity of the EM data to the target. The developed method provides an effective model-based interpolation/extrapolation tool for electromagnetic field data. This paper demonstrates that redatuming can be used for designing the optimized CSEM survey configuration. The numerical examples, for the Kevin Dome Electromagnetic Project Site, illustrate the practical effectiveness of the developed method.
\end{abstract}

(c) 2016 Elsevier B.V. All rights reserved.

\section{Introduction}

The land controlled-source electromagnetic (CSEM) surveys have been widely used in mineral exploration (Zhdanov, 2009, 2010). During the last decade, we have observed also a growing interest in an application of the marine version of this method to identifying oil- and gasbearing reservoirs (Constable, 2010). Another emerging technique is based on using the borehole transmitter and a grid of the surface receivers for detailed mapping of the subsurface resistivity of the oiland gas-producing fields (He et al., 2005, 2010). This method is often called Borehole-to-Surface Electromagnetic (BSEM) surveying. For example, a successful pilot BSEM field survey was executed recently in Saudi Arabia to identify oil- and water-bearing reservoir layers of a carbonate oil field water-injection zone (Marsala et al., 2011a, 2011b).

However, the target of the CSEM survey (e.g., BSEM), may be located deep underground, which may result in a relatively weak EM response in the receivers displayed on the surface of the earth. One way to overcome this problem is to move the receivers to the downhole beneath the overburden and closer to the reservoir target. However, this approach requires using downhole receivers, which is much more technically challenging and expensive than surface observations. In this paper we propose a numerical approach to estimate the EM field close to the estimated location of HC reservoir or other potential target. This approach is based on introducing the virtual receivers, located close to

\footnotetext{
* Corresponding author.

E-mail address: caihongzhu@hotmail.com (H. Cai).
}

the target, and redatuming the observed data from the actual receivers to the virtual one.

Another possible application of this approach is for solving the data interpolation and/or extrapolation problem. The modern method for interpretation of CSEM survey data is based on a 3D inversion of the observed data to estimate the subsurface conductivity distribution. The resolution of the recovered conductivity is significantly affected by the number of available data points on the observation surface, their spatial density and the size of the survey area covered by the actual receivers. One method to improve the model resolution of the inversion is to increase the number of receivers which requires significantly more effort. This paper develops a numerical method to determine the EM field in a much denser distributed virtual receiver covering the larger area of observation, if necessary.

Our developed method is based on the ideas of analytical downward and upward continuation of EM field between the observation surface and the surface located closer to a potential target. The principles of analytical continuation were originally introduced for the transformation of potential field data, and later on extended for the analytical continuation of electromagnetic and seismic field data by Zhdanov (Zhdanov, 1988). During the last decades the similar ideas became used in seismic exploration, where they appeared in the form of "redatuming" of seismic data, or seismic interferometry (Bakulin and Calvert, 2006; Schuster and Zhou, 2006; Schuster, 2009; Wapenaar et al., 2010). Recently, the same ideas were re-introduced for EM field continuation under the name of" electromagnetic interferometry" (Hunziker et al., 2009; Wapenaar et al., 2008). In this paper we demonstrate that the theory of analytical continuation of the EM field based on classical 
Stratton-Chu type integrals can be effectively used for solving this problem.

The developed EM redatuming theory and method may find a wide application in the EM field modeling, interpretation, survey design, interpolation, and extrapolation. In some special case, the method can be treated as a model-based interpolation.

In this paper, as an example, we consider a typical BSEM survey to illustrate the basic theory of EM redatuming. We also demonstrate the effectiveness of this method for optimizing the survey configuration for a geoelectrical model of the Kevin Dome Project Site.

\section{Integral representations of the EM field in an inhomogeneous medium}

It was demonstrated by Zhdanov (Zhdanov, 1988), that the Lorentz lemma can be used for deriving the integral representations of the EM fields in inhomogeneous medium similar to the Stratton-Chu formulas for a homogeneous medium (Berdichevsky and Zhdanov, 1984; Stratton, 1941). We will use here a similar approach to obtain integral representations of the EM field in an inhomogeneous medium.

We will consider a model with conductivity $\sigma$ and magnetic permeability $\mu$. We assume that the frequency-domain EM field $\{\mathbf{E}, \mathbf{H}\}$ in this model is excited by extraneous electric current, $\mathbf{j}^{\mathrm{Q}}$, distributed in some domain $Q$ (Fig. 1).

Let us assume that the conductivity $\sigma(\mathbf{r})$ can be described as a sum of the background conductivity, $\sigma_{b}(\mathbf{r})$, and anomalous conductivity, $\Delta \sigma(\mathbf{r})$, distributed within some domain $D$ :

$\sigma(\mathbf{r})=\left\{\begin{array}{l}\sigma_{b}(\mathbf{r})+\Delta \sigma(\mathbf{r}), \mathbf{r} \in D \\ \sigma_{b}(\mathbf{r}), \mathbf{r} \in C D\end{array}\right.$

where domain $C D$ is a complement of the bounded domain $D$ in the entire space.

The electromagnetic field in this model can be represented as a sum of the background and anomalous fields:

$\mathbf{E}=\mathbf{E}^{b}+\mathbf{E}^{a}, \mathbf{H}=\mathbf{H}^{b}+\mathbf{H}^{a}$

where the background field satisfies the following equations:

$\nabla \times \mathbf{H}^{b}=\sigma_{b} \mathbf{E}^{b}+\mathbf{j}^{Q}$,

$\nabla \times \mathbf{E}^{b}=i \omega \mu \mathbf{H}^{b}$,

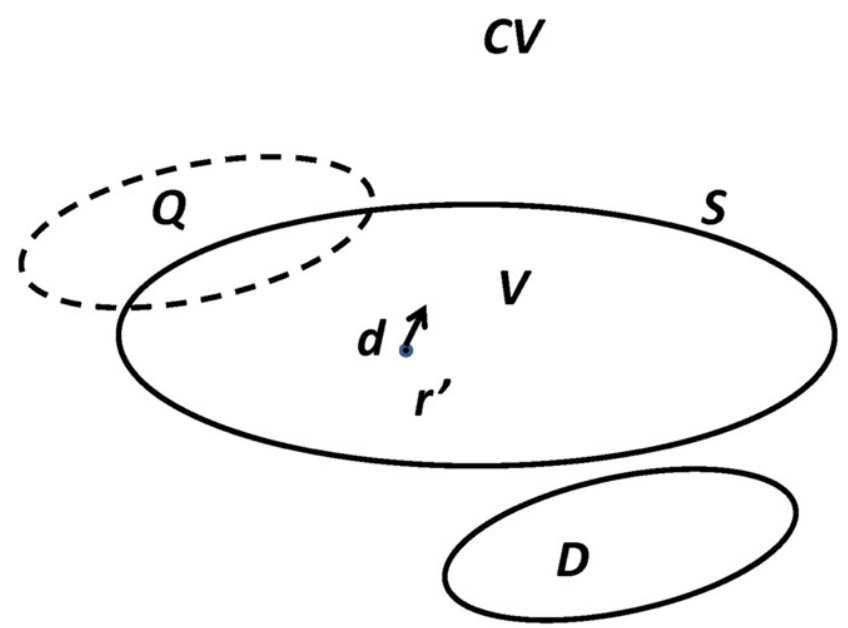

Fig. 1. Integral representations of the EM field inside domain $V$ of an inhomogeneous medium. Domain $C V$ is a complement of the bounded domain $V$ in the entire space. and the anomalous field is due to the induced current, $\mathbf{j}^{D}$, in the anomalous domain $D$ :

$\nabla \times \mathbf{H}^{a}=\sigma_{b} \mathbf{E}^{a}+\mathbf{j}^{D}$

$\nabla \times \mathbf{E}^{a}=i \omega \mu \mathbf{H}^{a}$,

where

$\mathbf{j}^{D}=\Delta \sigma \mathbf{E}$

We introduce arbitrary domain $V$ bounded by the surface $S$. We assume that the domain with anomalous conductivity is located outside domain $V: D \subset C V$. Our goal is to find the anomalous EM field in some point $\mathbf{r}$ inside domain $V$ from the known values of this field on the boundary $S$.

In order to solve this problem, we introduce two auxiliary background EM fields, $\left\{\mathbf{E}^{e}, \mathbf{H}^{e}\right\}$ and $\left\{\mathbf{E}^{m}, \mathbf{H}^{m}\right\}$. We assume that the EM field $\left\{\mathbf{E}^{e}, \mathbf{H}^{e}\right\}$ is generated by electric dipoles with unit moments $\mathbf{d}_{e}$, located at point with the radius-vector $\mathbf{r}^{\prime}$,

$\mathbf{j}^{e}=\mathbf{d}_{e} \delta\left(\mathbf{r}-\mathbf{r}^{\prime}\right)$

where $\delta$ is a delta function, and there are no magnetic-type sources. We will call the field, $\left\{\mathbf{E}^{e}, \mathbf{H}^{e}\right\}$, a background field of the electric type. It is a function of the observation point $\mathbf{r}$ and a location of the source, $\mathbf{r}$ :

$\mathbf{E}^{e}=\mathbf{E}^{e}\left(\mathbf{r}^{\prime} \mid \mathbf{r}\right), \mathbf{H}^{e}=\mathbf{H}^{e}\left(\mathbf{r}^{\prime} \mid \mathbf{r}\right)$

It can be demonstrated that this field is nothing else but the scalar product of vector $\mathbf{d}_{e}$ and the electric type Green's tensor, $\widehat{\mathbf{G}}_{E}^{e}$ and $\widehat{\mathbf{G}}_{H}^{e}$, for an inhomogeneous medium (Zhdanov, 1988, 2009):

$\mathbf{E}^{e}=\mathbf{d}_{e} \cdot \widehat{\mathbf{G}}_{E}^{e}, \mathbf{H}^{e}=\mathbf{d}_{e} \cdot \widehat{\mathbf{G}}_{H}^{e}$

At the same time, the auxiliary background EM field, $\left\{\mathbf{E}^{m}, \mathbf{H}^{m}\right\}$, is generated by magnetic dipole with unit moment $\mathbf{d}_{m}$, located at point with the radius-vector $\mathbf{r}^{\prime}$,

$\mathbf{j}^{m}=\mathbf{d}_{m} \delta\left(\mathbf{r}-\mathbf{r}^{\prime}\right)$

and we assume that there are no electric-type sources. We will call the field $\left\{\mathbf{E}^{m}, \mathbf{H}^{m}\right\}$ a background field of magnetic type:

$\mathbf{E}^{m}=\mathbf{E}^{m}\left(\mathbf{r}^{\prime} \mid \mathbf{r}\right), \mathbf{H}^{m}=\mathbf{H}^{m}\left(\mathbf{r}^{\prime} \mid \mathbf{r}\right)$

This field is in fact a scalar product of vector $\mathbf{d}_{m}$ and the magnetic type Green's tensor, $\widehat{\mathbf{G}}_{E}^{m}$ and $\widehat{\mathbf{G}}_{H}^{m}$, for an inhomogeneous medium (Zhdanov, 1988, 2009):

$\mathbf{E}^{m}=\mathbf{d}_{m} \cdot \widehat{\mathbf{G}}_{E}^{m}, \mathbf{H}^{m}=\mathbf{d}_{m} \cdot \widehat{\mathbf{G}}_{H}^{m}$.

The generalized Lorentz lemma describes the relationship between the electromagnetic field $\left\{\mathbf{E}^{A}, \mathbf{H}^{A}\right\}$ which is excited by the source $\left\{\mathbf{j}_{A}^{e}, \mathbf{j}_{A}^{m}\right\}$ and the electromagnetic field $\left\{\mathbf{E}^{B}, \mathbf{H}^{B}\right\}$ which is excited by the source $\left\{\mathbf{j}_{B}^{e}, \mathbf{j}_{B}^{m}\right\}$ in frequency domain (a detailed derivation can be found in the Appendix A):

$$
\begin{aligned}
\iint_{S}\{ & {\left.\left[\mathbf{E}^{B} \times \mathbf{H}^{A}\right]-\left[\mathbf{E}^{A} \times \mathbf{H}^{B}\right]\right\} \cdot d \mathbf{s} } \\
= & \iiint_{V}\left[i \omega \Delta \mu \mathbf{H}^{A} \cdot \mathbf{H}^{B}-\Delta \widetilde{\widetilde{\sigma}} \mathbf{E}^{A} \cdot \mathbf{E}^{B}\right] \mathrm{dv} \\
& +\iiint_{V}\left[\mathbf{E}^{A} \cdot \mathbf{j}_{B}^{e}+\mathbf{H}^{B} \cdot \mathbf{j}_{A}^{m}-\mathbf{E}^{B} \cdot \mathbf{j}_{A}^{e}-\mathbf{H}^{A} \cdot \mathbf{j}_{B}^{m}\right] \mathrm{dv},
\end{aligned}
$$


where

$\Delta \widetilde{\sigma}=\widetilde{\sigma}_{A}-\widetilde{\sigma}_{B}, \Delta \mu=\mu_{A}-\mu_{B}$

We can apply now generalized Lorentz lemma in Eq. (11) to the anomalous field, $\left\{\mathbf{E}^{a}, \mathbf{H}^{a}\right\}$, and the background field of the electric type, $\left\{\mathbf{E}^{e}, \mathbf{H}^{e}\right\}$ by letting $\mathbf{E}^{B}=\mathbf{E}^{e}, \mathbf{H}^{B}=\mathbf{H}^{e}, \mathbf{E}^{A}=\mathbf{E}^{a}$, and $\mathbf{H}^{A}=\mathbf{H}^{a}$.

Taking into account that there is no anomalous conductivity within domain $V(\Delta \sigma=0), \mathbf{j}_{A}^{m}=0, \mathbf{j}_{A}^{e}=\Delta \sigma \mathbf{E}=0$, and $\mathbf{j}_{B}^{m}=0$ in this case, we arrive at the following integral representation of the electric anomalous field:

$$
\begin{gathered}
\iint_{S}\left\{\left[\mathbf{E}^{e}\left(\mathbf{r}^{\prime} \mid \mathbf{r}\right) \times \mathbf{H}^{a}(\mathbf{r})\right]-\left[\mathbf{E}^{a}(\mathbf{r}) \times \mathbf{H}^{e}\left(\mathbf{r}^{\prime} \mid \mathbf{r}\right)\right]\right\} \cdot d \mathbf{s} \\
\quad=\iiint_{V}\left[\mathbf{E}^{a}(\mathbf{r}) \cdot \mathbf{d}_{e} \delta\left(\mathbf{r}-\mathbf{r}^{\prime}\right)\right] d v=\mathbf{d}_{e} \cdot \mathbf{E}^{a}\left(\mathbf{r}^{\prime}\right) .
\end{gathered}
$$

In a similar way, by applying generalized Lorentz lemma in Eq. (11) to the anomalous field, $\left\{\mathbf{E}^{a}, \mathbf{H}^{a}\right\}$, and the background field of magnetic type, $\left\{\mathbf{E}^{m}, \mathbf{H}^{m}\right\}$, we obtain an integral representation for magnetic anomalous field:

$$
\begin{gathered}
\iint_{S}\left\{\left[\mathbf{E}^{m}\left(\mathbf{r}^{\prime} \mid \mathbf{r}\right) \times \mathbf{H}^{a}(\mathbf{r})\right]-\left[\mathbf{E}^{a}(\mathbf{r}) \times \mathbf{H}^{m}\left(\mathbf{r}^{\prime} \mid \mathbf{r}\right)\right]\right\} \cdot d \boldsymbol{s} \\
\quad=-\iiint_{V}\left[\mathbf{H}^{a}(\mathbf{r}) \cdot \mathbf{d}_{m} \delta\left(\mathbf{r}-\mathbf{r}^{\prime}\right)\right] d v=-\mathbf{d}_{m} \mathbf{H}^{a}\left(\mathbf{r}^{\prime}\right) .
\end{gathered}
$$

Substituting Eq. (8) into Eq. (12), after some algebra, we have:

$$
\mathbf{d}_{e} \cdot \iint_{S}\left\{\left[\hat{\mathbf{G}}_{E}^{e}\left(\mathbf{r}^{\prime} \mid \mathbf{r}\right) \times \mathbf{H}^{a}(\mathbf{r})\right]+\left[\hat{\mathbf{G}}_{H}^{e}\left(\mathbf{r}^{\prime} \mid \mathbf{r}\right)\right] \times \mathbf{E}^{a}(\mathbf{r})\right\} \cdot d \mathbf{s}=\mathbf{d}_{e} \cdot \mathbf{E}^{a}\left(\mathbf{r}^{\prime}\right) .
$$

We can obtain a similar representation for the magnetic field as well by substituting Eq. (10) into formula (13):

$$
\mathbf{d}_{m} \cdot \iint_{S}\left\{\left[\hat{\mathbf{G}}_{E}^{m}\left(\mathbf{r}^{\prime} \mid \mathbf{r}\right) \times \mathbf{H}^{a}(\mathbf{r})\right]+\left[\hat{\mathbf{G}}_{H}^{m}\left(\mathbf{r}^{\prime} \mid \mathbf{r}\right) \times \mathbf{E}^{a}(\mathbf{r})\right]\right\} \cdot d \mathbf{s}=-\mathbf{d}_{m} \cdot \mathbf{H}^{a}\left(\mathbf{r}^{\prime}\right) .
$$

Note that, the surface integrals in Eqs. (14) and (15) can be expressed by the Stratton-Chu type integrals for an inhomogeneous medium (Zhdanov, 1988), $\mathbf{S}_{E^{a}}\left(\mathbf{r}^{\prime}\right)$ and $\mathbf{S}_{H^{a}}\left(\mathbf{r}^{\prime}\right)$ :

$$
\begin{aligned}
& \mathbf{S}_{S}^{e}\left(\mathbf{r}^{\prime}\right)=\iint_{S}\left\{\left[\widehat{\mathbf{G}}_{E}^{e}\left(\mathbf{r}^{\prime} \mid \mathbf{r}\right) \times \mathbf{H}^{a}(\mathbf{r})\right]+\left[\widehat{\mathbf{G}}_{H}^{e}\left(\mathbf{r}^{\prime} \mid \mathbf{r}\right) \times \mathbf{E}^{a}(\mathbf{r})\right]\right\} \cdot d \mathbf{s}, \\
& \mathbf{S}_{S}^{m}\left(\mathbf{r}^{\prime}\right)=-\iint_{S}\left\{\left[\widehat{\mathbf{G}}_{E}^{m}\left(\mathbf{r}^{\prime} \mid \mathbf{r}\right) \times \mathbf{H}^{a}(\mathbf{r})\right]+\left[\widehat{\mathbf{G}}_{H}^{m}\left(\mathbf{r}^{\prime} \mid \mathbf{r}\right) \times \mathbf{E}^{a}(\mathbf{r})\right]\right\} \cdot d \mathbf{s} .
\end{aligned}
$$

Taking into account that Eqs. (14) and (15) hold for arbitrary vectors $\left\{\mathbf{d}_{e}, \mathbf{d}_{m}\right\}$ and that the Stratton-Chu type integrals are equal to zero outside domain $V$, we can rewrite these integral representations in the final form as follows:

$\mathbf{S}_{S}^{e}\left(\mathbf{r}^{\prime}\right)=\left\{\begin{array}{l}\mathbf{E}^{a}\left(\mathbf{r}^{\prime}\right), \mathbf{r}^{\prime} \in V \\ 0, \mathbf{r}^{\prime} \in C V\end{array} ;\right.$

$\mathbf{S}_{S}^{m}\left(\mathbf{r}^{\prime}\right)=\left\{\begin{array}{l}\mathbf{H}^{a}\left(\mathbf{r}^{\prime}\right), \mathbf{r}^{\prime} \in V \\ 0, \mathbf{r}^{\prime} \in C V\end{array}\right.$

As one can see, Eqs. (16) and (17) explicitly use the electric and magnetic Green's tensor, $\widehat{\mathbf{G}}_{E}^{e}, \widehat{\mathbf{G}}_{H}^{e}, \widehat{\mathbf{G}}_{E}^{m}$, and $\widehat{\mathbf{G}}_{H}^{m}$. In applications, it is more convenient to use the integral representations (12) and (13), based on the background electromagnetic fields of the electric and magnetic type $\left\{\mathbf{E}^{e}, \mathbf{H}^{e}\right\}$ and,$\left\{\mathbf{E}^{m}, \mathbf{H}^{m}\right\}$. It is important to emphasize that the auxiliary background field pairs can be based on inhomogeneous background conductivity rather than on a simple layered conductivity model because generalized Lorentz lemma holds for arbitrary background conductivity distribution.

\section{Redatuming problem for the BSEM survey configuration}

Let us consider a typical borehole-to-surface electromagnetic survey with the transmitter $T_{A}$ located at some point $A$ within the borehole and the receivers distributed over the earth surface $\Sigma$ at points with the radius-vector $\mathbf{r}^{\prime}$ (Fig. 2). Let us consider a horizontal plane $P$ located at a depth $z_{0}$ in the ground (with the axis $z$ directed downward). Note that point $A$ of the transmitter location could be above or below the horizontal plane $P$. We also assume that the conductivity of the earth between the surface of the earth $\Sigma$ and the horizontal plane $P$ is known and it is equal to the background conductivity $\sigma_{b}(\mathbf{r})$; however, below plane $P$ the conductivity $\sigma(\mathbf{r})$ is unknown and is characterized by some anomalous conductivity:

$\sigma(\mathbf{r})=\left\{\begin{array}{l}\sigma_{b}(\mathbf{r}), z<z_{0} \\ \sigma_{b}(\mathbf{r})+\Delta \sigma(\mathbf{r}), z>z_{0}\end{array}\right.$

Let us consider a spherical cap, $S_{R}$, in the upper half-space with a center located at the transmitter, point $A$, and a radius $R$. We will denote the domain bounded by this spherical cap and a part $P_{R}$, of the horizontal plane $P$, as $V_{R}$. Applying integral representation (12) to this domain, bounded by the closed surface $S_{R} \cup P_{R}$, we can write.

$\mathbf{d} \cdot \mathbf{E}^{a}\left(\mathbf{r}^{\prime}\right)=\iint_{S_{R} \cup P_{R}}\left\{\left[\mathbf{E}^{e}\left(\mathbf{r}^{\prime} \mid \mathbf{r}\right) \times \mathbf{H}^{a}(\mathbf{r})\right]-\left[\mathbf{E}^{a}(\mathbf{r}) \times \mathbf{H}^{e}\left(\mathbf{r}^{\prime} \mid \mathbf{r}\right)\right]\right\}$.

In the limit, $R \rightarrow \infty$, the surface integral over spherical cap $S_{R}$ will go to zero due to Sommerfeld radiation conditions (Zhdanov, 1988), and we will have in the last formula an integral over the horizontal plane $P$ only:

$\mathbf{d} \cdot \mathbf{E}^{a}\left(\mathbf{r}^{\prime}\right)=\iint_{P}\left\{\left[\mathbf{E}^{e}\left(\mathbf{r}^{\prime} \mid \mathbf{r}\right) \times \mathbf{H}^{a}(\mathbf{r})\right]-\left[\mathbf{E}^{a}(\mathbf{r}) \times \mathbf{H}^{e}\left(\mathbf{r}^{\prime} \mid \mathbf{r}\right)\right]\right\} \cdot d \mathbf{s}$.

We can obtain a similar expression for the magnetic field as well:

$\mathbf{d} \cdot \mathbf{H}^{a}\left(\mathbf{r}^{\prime}\right)=-\iint_{P}\left\{\left[\mathbf{E}^{m}\left(\mathbf{r}^{\prime} \mid \mathbf{r}\right) \times \mathbf{H}^{a}(\mathbf{r})\right]-\left[\mathbf{E}^{a}(\mathbf{r}) \times \mathbf{H}^{m}\left(\mathbf{r}^{\prime} \mid \mathbf{r}\right)\right]\right\} \cdot$

Note that, in the last formulas $\left\{\mathbf{E}^{a}(\mathbf{r}), \mathbf{H}^{a}(\mathbf{r})\right\}$ is an anomalous EM field, on the horizontal plane $P$, generated by transmitter $T_{A}$ for the model with given anomalous conductivity; $\left\{\mathbf{E}^{e}\left(\mathbf{r} \mid \mathbf{r}^{\prime}\right), \mathbf{H}^{e}\left(\mathbf{r} \mid \mathbf{r}^{\prime}\right)\right\}$ is a background EM field generated in the medium with background conductivity $\sigma_{b}$ by an electric dipole with the unit moment $\mathbf{d}$, located at a point with the radius-vectors $\mathbf{r}^{\prime}$; and $\left\{\mathbf{E}^{m}\left(\mathbf{r} \mid \mathbf{r}^{\prime}\right), \mathbf{H}^{m}\left(\mathbf{r} \mid \mathbf{r}^{\prime}\right)\right\}$ is a background EM field generated in the medium with background conductivity $\sigma_{b}$ by a magnetic dipole with the unit moment $\mathbf{d}$, located at a point with the radius-vectors r'. As we can see that by introducing the auxiliary

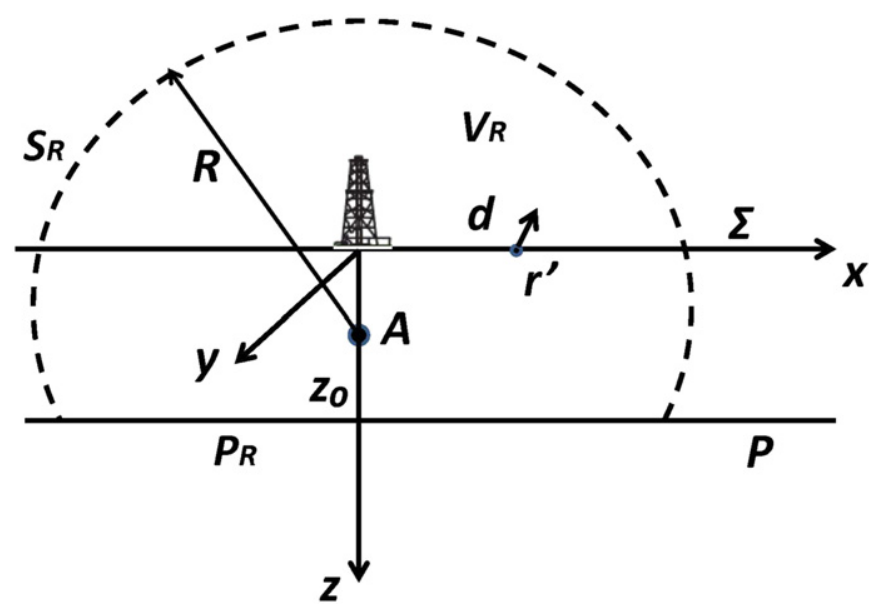

Fig. 2. A model of a typical borehole-to-surface electromagnetic survey with the transmitter $T_{A}$ located at some point $A$ within the borehole and the receivers distributed over the earth's surface $\Sigma$ at points with the radius-vector $\mathbf{r}$. 


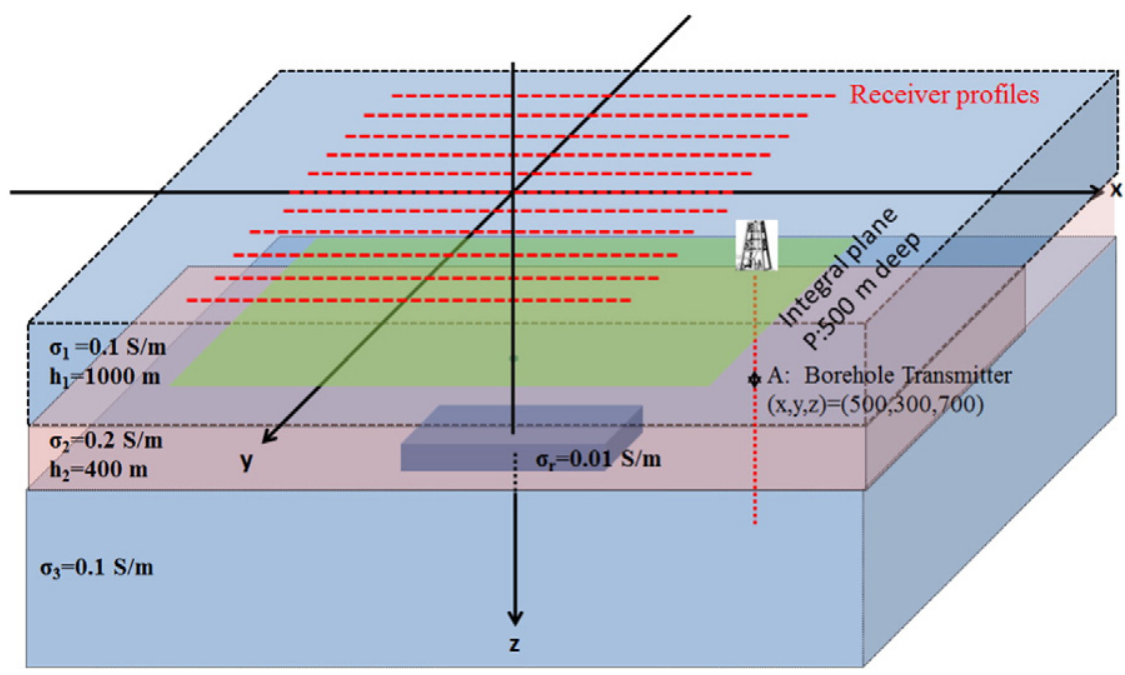

Fig. 3. 3D view of the synthetic BSEM model.

background field, the Green's tensor in Eqs. (16) and (17) can be eliminated.

Eqs. (22) and (23) make it possible to calculate the anomalous field $\left\{\mathbf{E}^{a}\left(\mathbf{r}^{\prime}\right), \mathbf{H}^{a}\left(\mathbf{r}^{\prime}\right)\right\}$ at any point on the surface $\Sigma$ if we know this field on the horizontal plane $P$. Using the Stratton-Chu formulas (16) through (19), we can rewrite Eqs. (22) and (23) in equivalent form as follows:

$$
\begin{aligned}
\mathbf{E}^{a}\left(\mathbf{r}^{\prime}\right) & =\mathbf{S}_{P}^{e}\left(\mathbf{r}^{\prime}\right) \\
& =\iint_{S}\left\{\widehat{\mathbf{G}}_{E}^{e}\left(\mathbf{r}^{\prime} \mid \mathbf{r}\right) \cdot\left[\mathbf{H}_{\tau}^{a}(\mathbf{r}) \times \mathbf{n}\right]+\widehat{\mathbf{G}}_{H}^{e}\left(\mathbf{r}^{\prime} \mid \mathbf{r}\right) \cdot\left[\mathbf{E}_{\tau}^{a}(\mathbf{r}) \times \mathbf{n}\right]\right\} d s, \\
\mathbf{H}^{a}\left(\mathbf{r}^{\prime}\right) & =\mathbf{S}_{P}^{m}\left(\mathbf{r}^{\prime}\right) \\
& =-\iint_{S}\left\{\widehat{\mathbf{G}}_{E}^{m}\left(\mathbf{r}^{\prime} \mid \mathbf{r}\right) \cdot\left[\mathbf{H}_{\tau}^{a}(\mathbf{r}) \times \mathbf{n}\right]+\widehat{\mathbf{G}}_{H}^{m}\left(\mathbf{r}^{\prime} \mid \mathbf{r}\right) \cdot\left[\mathbf{E}_{\tau}^{a}(\mathbf{r}) \times \mathbf{n}\right]\right\} d s
\end{aligned}
$$

where we took into account that $d \mathbf{s}=\mathbf{n} d s$, and $\mathbf{n}$ is a unit vector of the normal to the surface $P$ directed downward, and $\mathbf{E}_{\tau}^{P a}, \mathbf{H}_{\tau}^{P a}$ are the tangential components of the anomalous field on the surface $P$.

Eqs. (24) and (25) can be used for redatuming of anomalous EM field data. These equations transform the anomalous EM field from the underground surface $P$ to the observation surface $\Sigma$. The same equation can be used for downward analytical continuation of the EM field from the observation surface $\Sigma$ to the horizontal plane $P$ located at a depth $z_{0}$. In this case, Stratton-Chu integral formulas (24) and (25) should be treated as integral equations with respect to the unknown EM field $\left\{\mathbf{E}_{\tau}^{P a}, \mathbf{H}_{\tau}^{P a}\right\}$.

\section{Regularized conjugate method of solving the Stratton-Chu integral equations}

Different methods of the downward analytical continuation of the EM field were discussed in Zhdanov (Zhdanov, 1988). In this paper we have developed a technique for solving this problem based on the
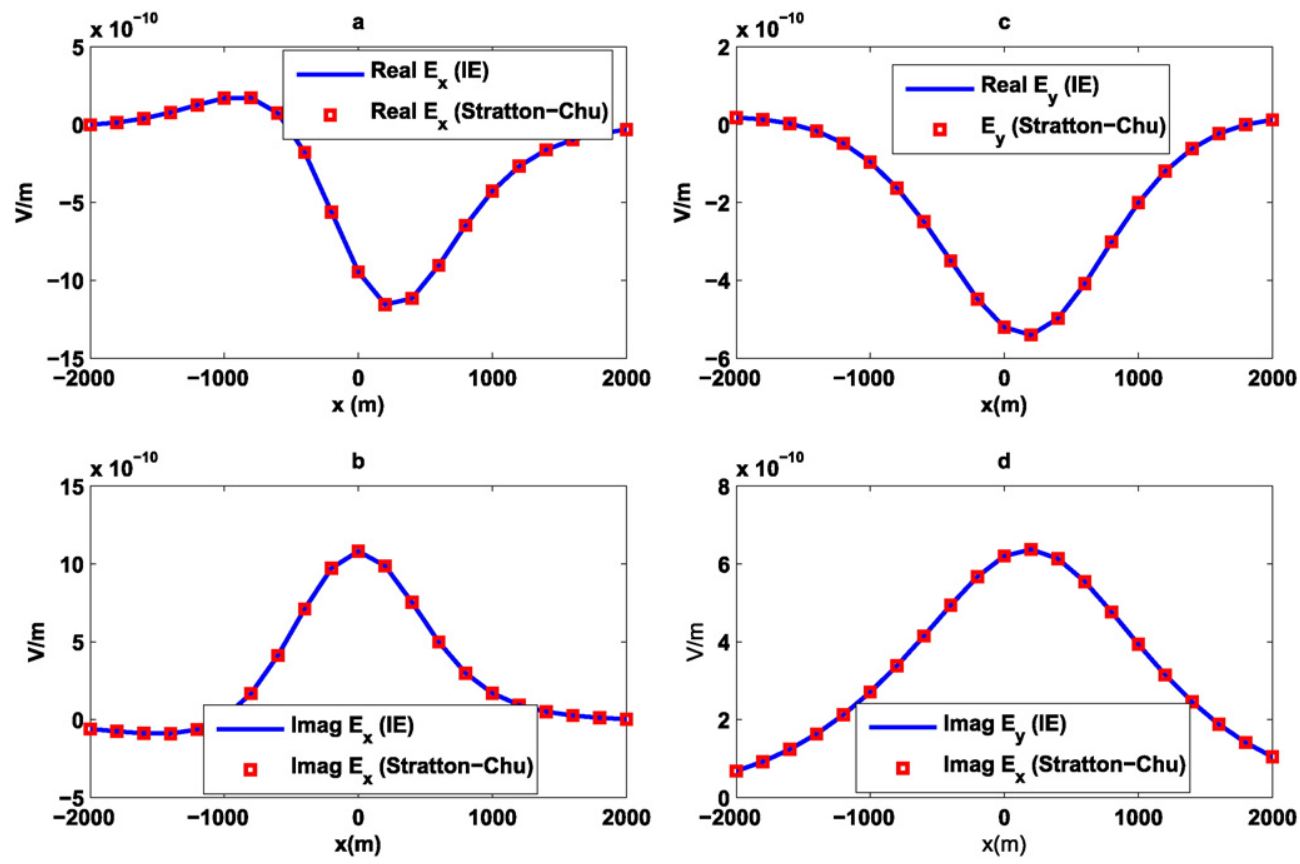

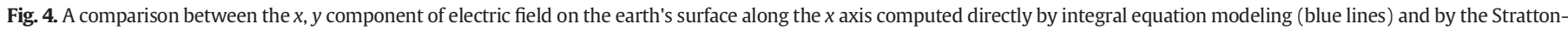

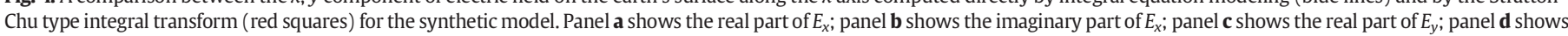
the imaginary part of $E_{y}$. 

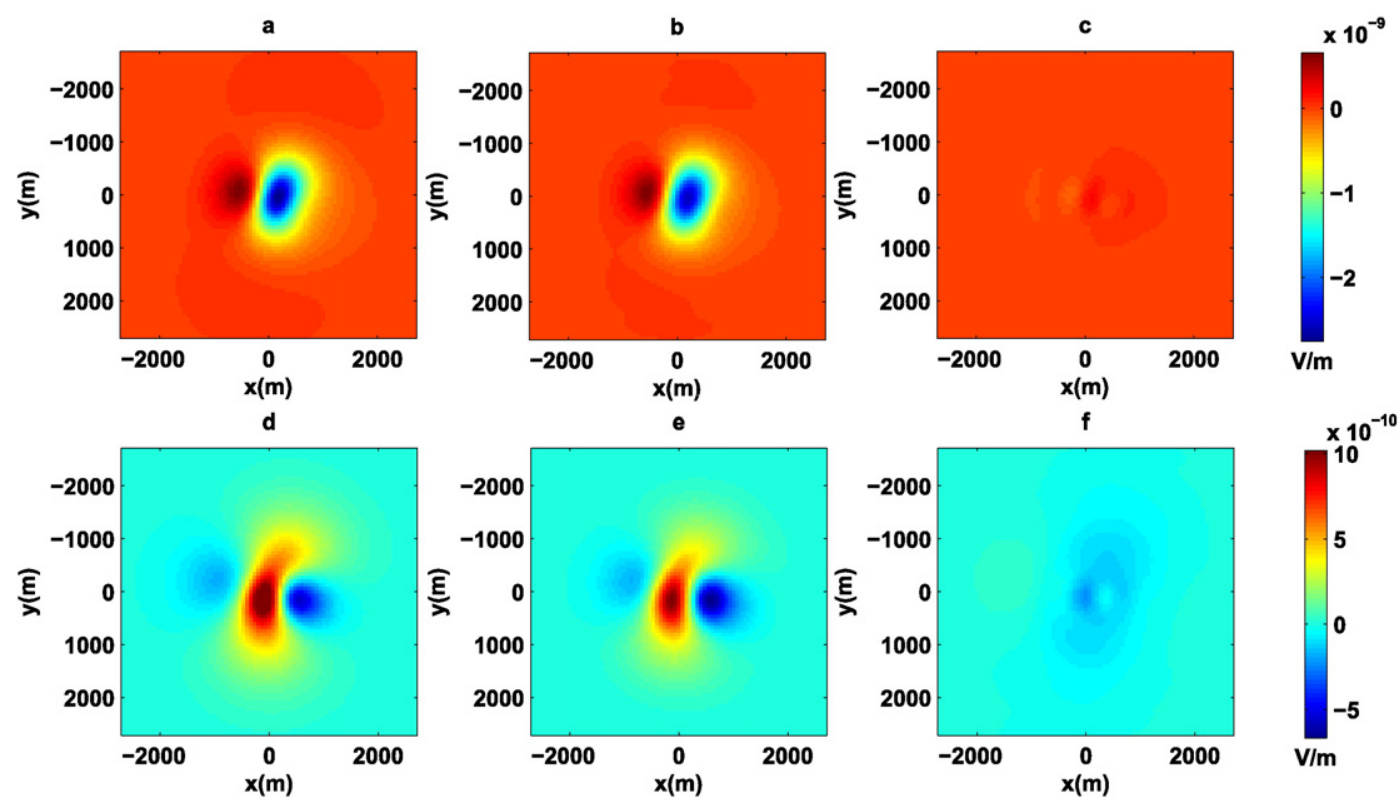

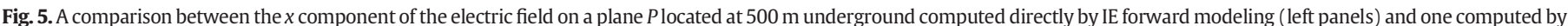

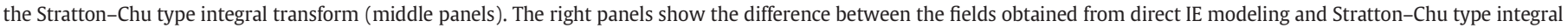
transform. Panels $\mathbf{a}, \mathbf{b}$, and $\mathbf{c}$ show the real parts of $E_{x}$; while panels $\mathbf{d}, \mathbf{e}$ and $\mathbf{f}$ show the imaginary parts of $E_{x}$.

regularized conjugate gradient (RCG) method. In the framework of this approach, the Stratton-Chu integral in Eqs. (24) and (25) can be written as follows:

$\left[\begin{array}{l}\mathbf{E}^{\Sigma a} \\ \mathbf{H}^{\Sigma a}\end{array}\right]=\left[\begin{array}{ll}G_{H}^{e} & G_{E}^{e} \\ G_{H}^{m} & G_{E}^{m}\end{array}\right]\left[\begin{array}{l}\mathbf{E}_{\tau}^{P a} \\ \mathbf{H}_{\tau}^{P a}\end{array}\right]$,

or in compact form:

$\mathbf{M}^{\Sigma}=\mathcal{G} \mathbf{M}^{P}$

where $\mathbf{M}^{\Sigma}$ and $\mathbf{M}^{P}$, represent the anomalous EM field given on the
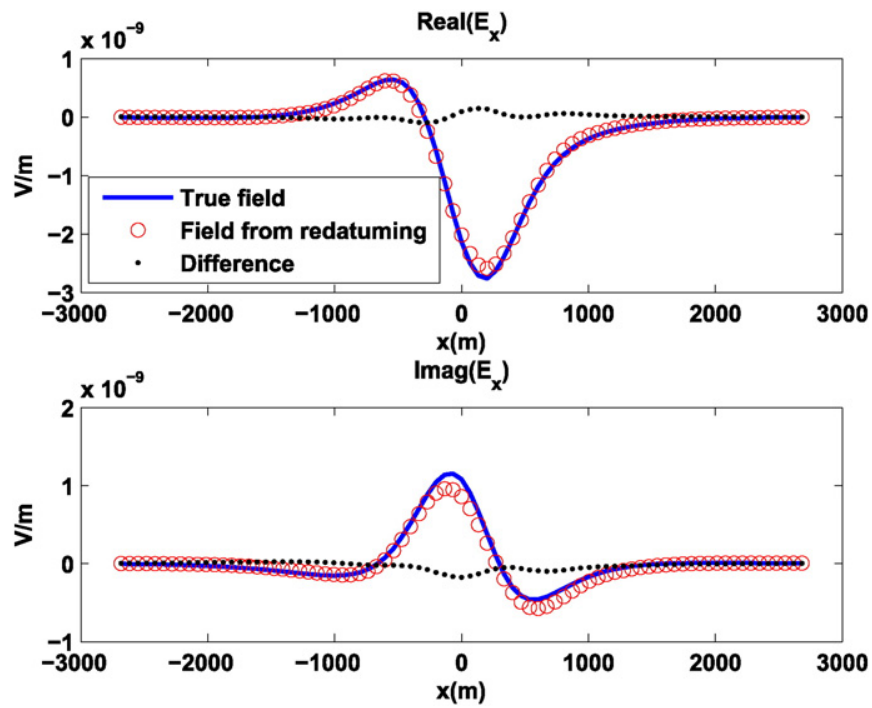

Fig. 6. A comparison between the $x$ component of the electric field on a plane $P$ located at $500 \mathrm{~m}$ underground computed directly by IE forward modeling (solid blue) and one computed by the Stratton-Chu type integral transform or redatuming (red circles) at $y=0$. The black dots show the difference between the field computed from IE modeling and Stratton-Chu type integral transform. The upper panel shows the real part while the lower panel represents the imaginary part of the field. surfaces $\Sigma$ and $P$, respectively:

$\mathbf{M}^{\Sigma}=\left[\mathbf{E}^{\Sigma a}, \mathbf{H}^{\Sigma a}\right]^{T}, \mathbf{M}^{P}=\left[\mathbf{E}_{\tau}^{P a}, \mathbf{H}_{\tau}^{P a}\right]^{T}$

matrix operator $\mathcal{G}$ is defined by the following matrix:

$\mathcal{G}=\left[\begin{array}{ll}\mathbf{G}_{H}^{e} & \mathbf{G}_{E}^{e} \\ \mathbf{G}_{H}^{m} & \mathbf{G}_{E}^{m}\end{array}\right]$

and $\mathbf{G}_{E, H}^{e}$ and $\mathbf{G}_{E, H}^{m}$ are the corresponding Green's matrix operators:

$\mathbf{G}_{E, H}^{e} \mathbf{f}=\iint_{S} \widehat{\mathbf{G}}_{E, H}^{e}\left(\mathbf{r}^{\prime} \mid \mathbf{r}\right) \cdot[\mathbf{f} \times \mathbf{n}] d s$,

$\mathbf{G}_{E, H}^{m} \mathbf{f}=-\iint_{S} \widehat{\mathbf{G}}_{E, H}^{m}\left(\mathbf{r}^{\prime} \mid \mathbf{r}\right) \cdot[\mathbf{f} \times \mathbf{n}] d s$.

Expressions (26) and (27) represent a compact form of the StrattonChu integral equation, which can be used for redatuming of the anomalous EM field data.

The integral equation (27) is ill posed; the solution can be nonunique and unstable. In order to obtain a stable solution, we consider a minimization of the Tikhonov parametric functional (Zhdanov, 2002):

$$
\begin{aligned}
P^{\alpha}\left(\mathbf{m}, \mathbf{M}^{\Sigma}\right)= & \left(\mathcal{G}(\mathbf{m})-\mathbf{M}^{\Sigma}\right)^{*}\left(\mathcal{G}(\mathbf{m})-\mathbf{M}^{\Sigma}\right) \\
& +\alpha\left(\mathbf{m}-\mathbf{m}_{\text {apr }}\right)^{*}\left(\mathbf{m}-\mathbf{m}_{\text {apr }}\right) \rightarrow \min ,
\end{aligned}
$$

where asterisk" denotes a complex conjugate transposed matrix, and $\mathbf{m}_{a p r}$ is some a priori vector of the EM field at the surface $P$.

We apply the regularized conjugate gradient method (RCG) to solve the minimization problem of (28) (Zhdanov, 2002).

\section{Synthetic model studies}

In this section we consider an application of the developed method for redatuming the data for the borehole-to-surface electromagnetic survey, introduced above (see Fig. 2). A 3D view of the synthetic BSEM model is shown in Fig. 3. This model consists of a near-surface one km thick layer with the conductivity $\sigma_{1}=0.1 \mathrm{~S} / \mathrm{m}$, the second layer having the conductivity $\sigma_{2}=0.2 \mathrm{~S} / \mathrm{m}$ and a 
a

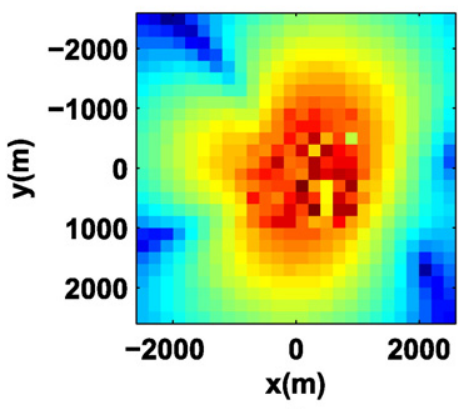

c

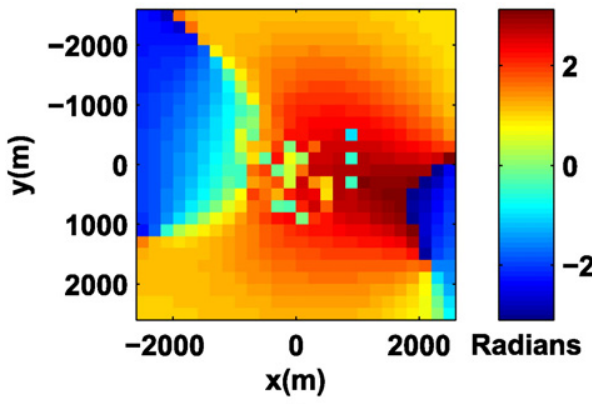

e
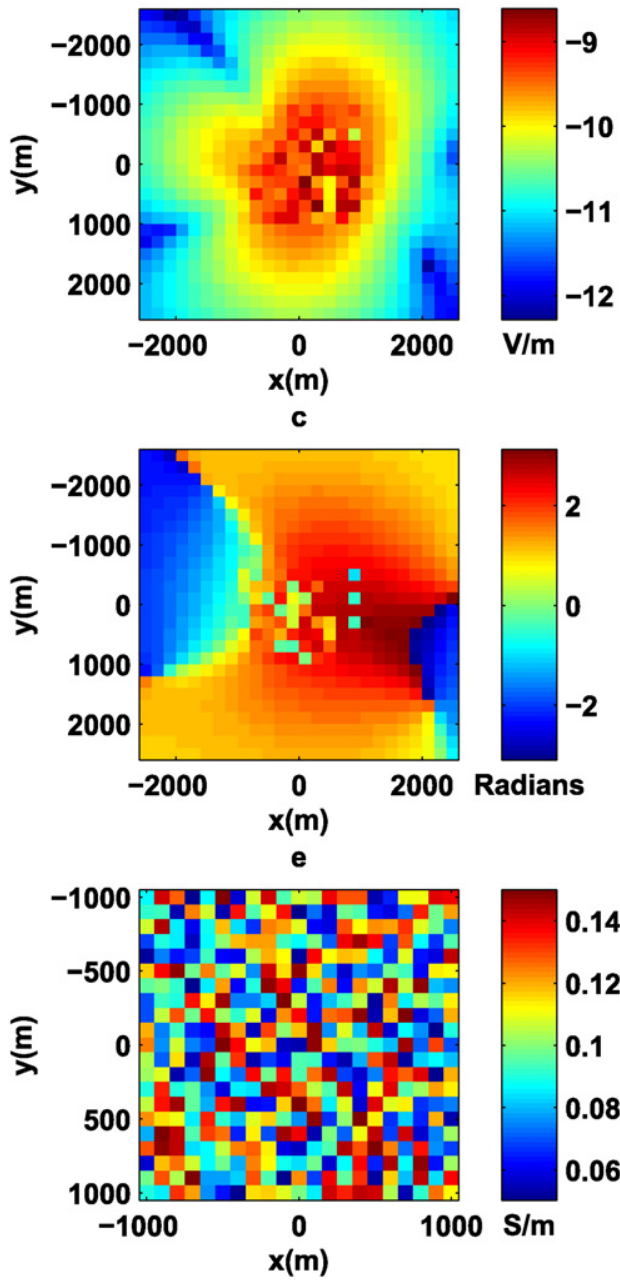

b

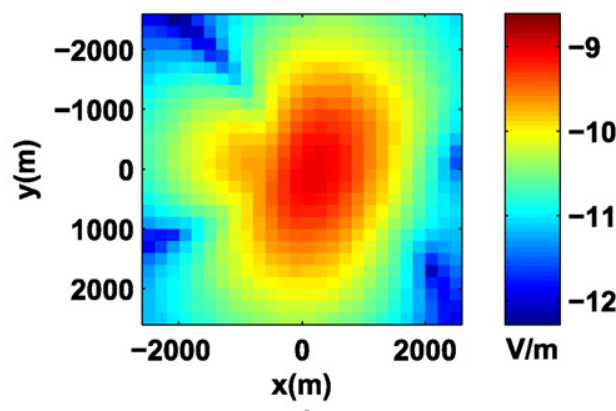

d
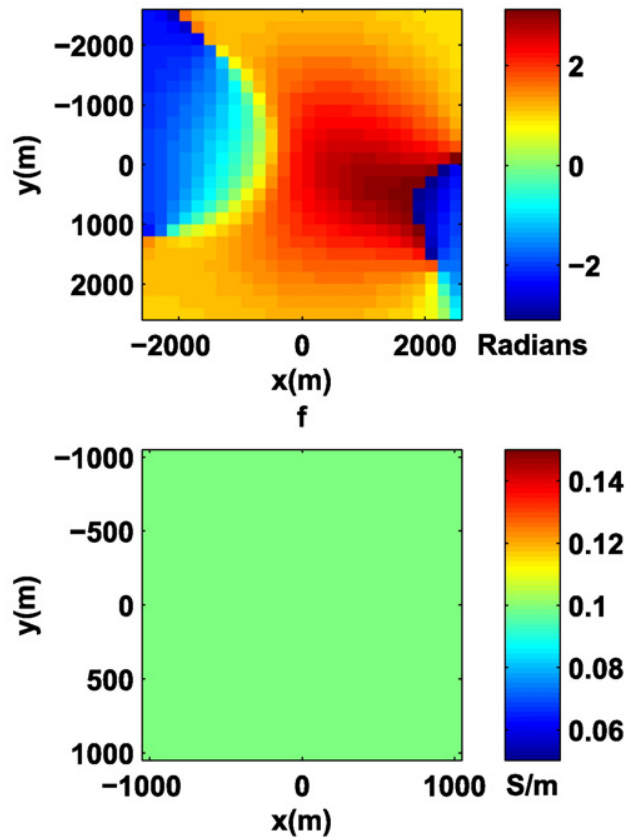

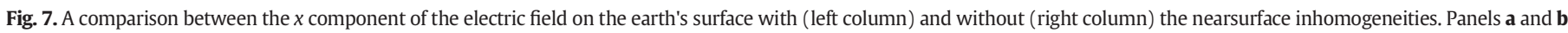

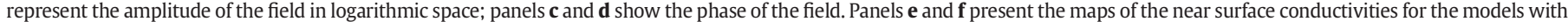
(left column) and without (right column) the nearsurface distortions.

a
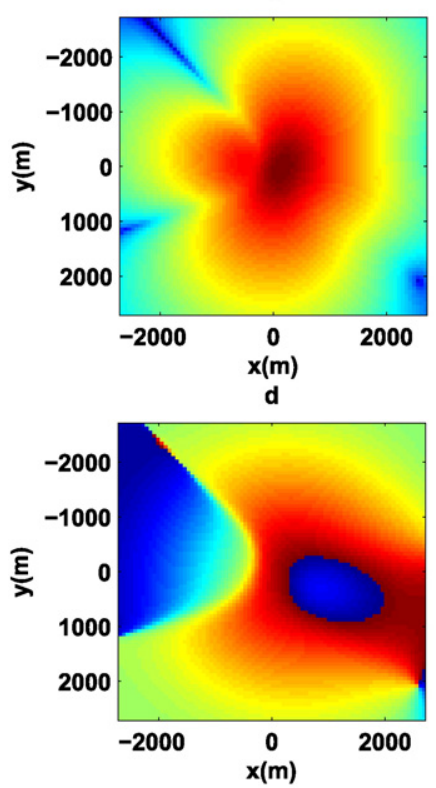

b

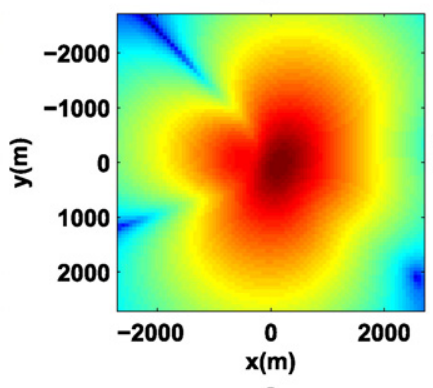

e

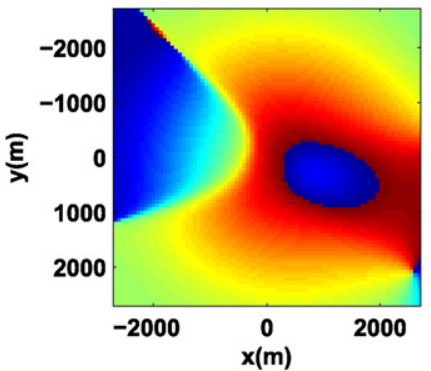

c

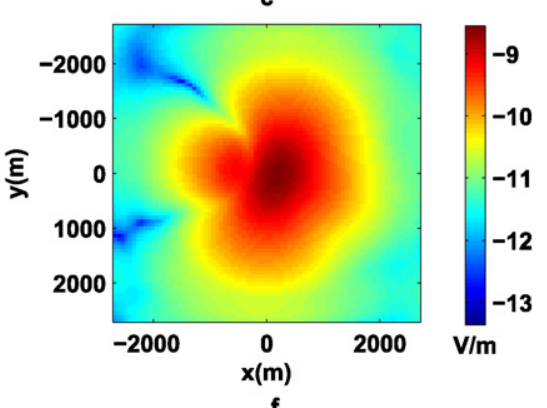

f

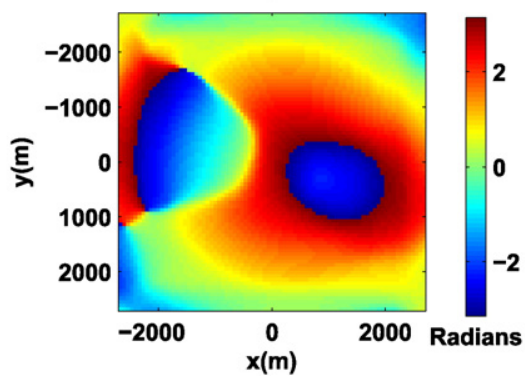

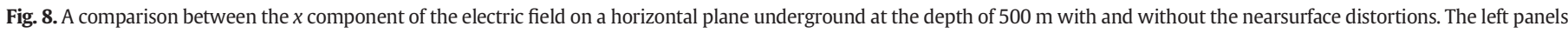

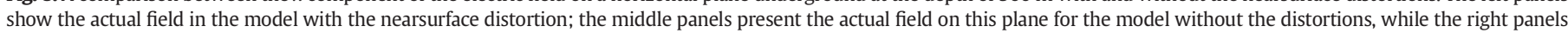
show the field obtained by redatuming. Panels $\mathbf{a}, \mathbf{b}$ and $\mathbf{c}$ represent the amplitude of the field in logarithmic space; panels $\mathbf{d}, \mathbf{e}$ and $\mathbf{f}$ show the phase of the field. 


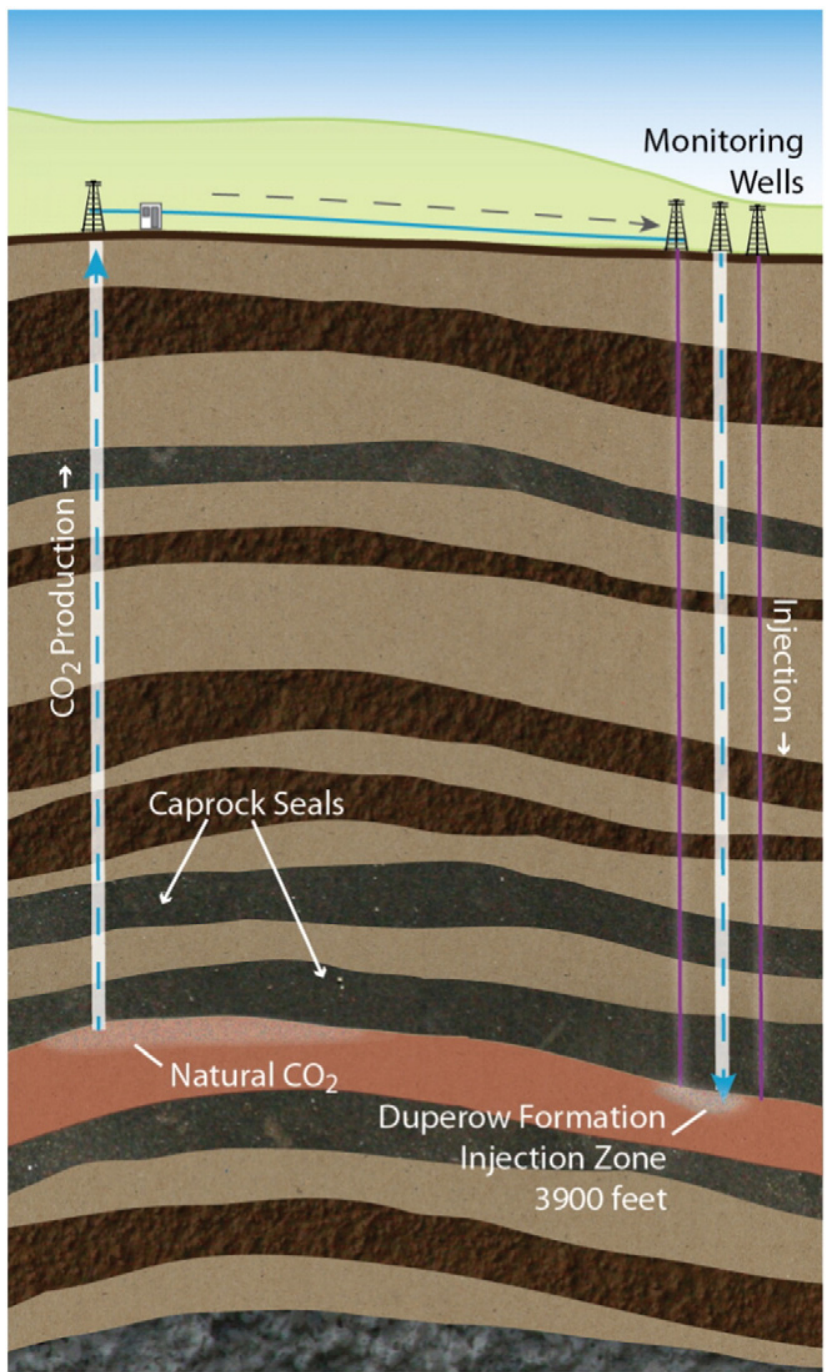

Fig. 9. Vertical section for Kevin Dome EM project site.

thickness of $400 \mathrm{~m}$, underlaid by a homogeneous half-space with the conductivity $\sigma_{3}=0.1 \mathrm{~S} / \mathrm{m}$. A resistive hydrocarbon (HC) reservoir layer with the conductivity of $0.01 \mathrm{~S} / \mathrm{m}$ is located within the second layer as shown in Fig. 3. The frequency domain EM field in this model at a frequency of $5 \mathrm{~Hz}$ is excited by a vertical electric dipole transmitter, located at a depth of $700 \mathrm{~m}$ in the vertical borehole, as shown in Fig. 3. For simplicity, we will not consider borehole casing for this and the following BSEM model. We have conducted a few numerical experiments for this model.

In the first numerical example, we assume that the EM field is observed in the set of virtual receivers located on the horizontal plane $P$ at a depth of $500 \mathrm{~m}$ within the first layer (see Fig. 3). The goal is to transform the anomalous EM field upward from the horizontal plane $P$ to the surface of the earth $\Sigma$

In order to solve this problem, we can simplify formulas (22) and (23) for the case where $P$ is a horizontal plane. For example, the equation for the components $E_{\beta}^{a}(\beta=x, y, z)$ of the anomalous electric field takes the following form:

$$
\begin{aligned}
E_{\beta}^{a}\left(\mathbf{r}^{\prime}\right)= & \iint_{P}\left\{E_{x}^{\beta}\left(\mathbf{r} \mid \mathbf{r}^{\prime}\right) H_{y}^{a}(\mathbf{r})-E_{y}^{\beta}\left(\mathbf{r} \mid \mathbf{r}^{\prime}\right) H_{x}^{a}(\mathbf{r})\right\} d x d y \\
& +\iint_{P}\left\{H_{x}^{\beta}\left(\mathbf{r} \mid \mathbf{r}^{\prime}\right) E_{y}^{a}(\mathbf{r})-H_{y}^{\beta}\left(\mathbf{r} \mid \mathbf{r}^{\prime}\right) E_{x}^{a}(\mathbf{r})\right\} d x d y
\end{aligned}
$$

where $\beta=x, y, z$, and the auxiliary EM field, $\left\{\mathbf{E}^{\beta}, \mathbf{H}^{\beta}\right\}$, is generated in a medium with the background conductivity $\sigma_{b}$ by an electric dipole with the unit moment $\mathbf{d}_{\beta}$, located at a point with the radius-vectors $\mathbf{r}$ :

$\mathbf{j}^{\beta}=\mathbf{d}_{\beta} \delta\left(\mathbf{r}-\mathbf{r}^{\prime}\right), \mathbf{r}^{\prime} \in \Sigma$.

In practice, the observed data are collected in the discrete grid of the receivers. Therefore, we have to write a discrete form of Eq. (29), which can be expressed as follows:

$$
\begin{aligned}
E_{\beta}^{a}\left(\mathbf{r}^{\prime}\right)= & \lambda^{2} \sum_{n=-N}^{N} \sum_{l=-L}^{L}\left\{E_{x}^{\beta}\left(n \Delta x_{\lambda}, l \Delta y_{\lambda} \mid \mathbf{r}^{\prime}\right) H_{y}^{a}\left(n \Delta x_{\lambda}, l \Delta y_{\lambda}\right)\right. \\
& \left.-E_{y}^{\beta}\left(n \Delta x_{\lambda}, l \Delta y_{\lambda} \mid \mathbf{r}^{\prime}\right) H_{x}^{a}\left(n \Delta x_{\lambda}, l \Delta y_{\lambda}\right)\right\} \Delta x_{\lambda} \Delta y_{\lambda} \\
& +\lambda^{2} \sum_{n=-N}^{N} \sum_{l=-L}^{L}\left\{H_{x}^{\beta}\left(n \Delta x_{\lambda}, l \Delta y_{\lambda} \mid \mathbf{r}^{\prime}\right) E_{y}^{a}\left(n \Delta x_{\lambda}, l \Delta y_{\lambda}\right)\right. \\
& \left.-H_{y}^{\beta}\left(n \Delta x_{\lambda}, l \Delta y_{\lambda} \mid \mathbf{r}^{\prime}\right) E_{x}^{a}\left(n \Delta x_{\lambda}, l \Delta y_{\lambda}\right)\right\} \Delta x_{\lambda} \Delta y_{\lambda},
\end{aligned}
$$

where $\lambda$ is a wavelength in the first layer, containing plane $P$; and $\Delta x_{\lambda}=$ $\Delta x / \lambda$ and $\Delta y_{\lambda}=\Delta y / \lambda$ are the dimensionless separations between the receivers.

Note that, for the model shown in Fig. 3, the background conductivity $\sigma_{b}$ is formed by the conductivities $\sigma_{1}, \sigma_{2}, \sigma_{3}$ of the three horizontal homogeneous layers, therefore, the auxiliary EM field, $\left\{\mathbf{E}^{\beta}, \mathbf{H}^{\beta}\right\}$, can be easily computed as the field generated in the three-layered background model.

We have also computed the anomalous EM field caused by the presence of the $\mathrm{HC}$ reservoir, both at the horizontal plane $P$ and at the surface of the earth $\Sigma$ in a set of the surface receivers using integral equation method (IE). Recall that, if the EM field on plane $P$ is given, we can also apply Eq. (31) which is the discretized form of StrattonChu type integral transform, to compute the field on the surface. Fig. 4 shows a comparison of anomalous electric field on the earth's surface along the $x$-axis (blue lines) directly computed from IE and from Stratton-Chu type integral transform. As one can see, the transformed data practically coincide with the observed data on the earth's surface which validated the mathematical formulation in our paper.

In the next numerical experiment we assumed that the EM field was observed in the set of receivers located on the earth's surface $\Sigma$, and we considered expression (31) as an equation with regard to the unknown values of the EM field in the horizontal plane $P$ at a depth of $500 \mathrm{~m}$ within the first layer (see Fig. 3). Note that the synthetic observed data were contaminated by $5 \%$ random noise. We applied the RCG algorithm, (Zhdanov, 2002) to solve this equation.

The left panels in Fig. 5 show the maps of the anomalous EM field on the horizontal plane $P$ obtained directly from integral equation modeling. For comparison, we present in the middle panels of Fig. 5 the similar maps produced by redatuming of anomalous EM field from the earth's surface to the horizontal plane $P$ and the right panels show the absolute difference. Fig. 6 shows a comparison between the field computed from direct forward modeling and redatuming on plane $P$ at $y=0$. From these two figures, one can see that the transformed EM field corresponds well to the results of the direct forward modeling. Note also that we have many more virtual receivers on the plane $P$ than the number of the actual receivers on the earth's surface., and significantly more dense distribution of the virtual receiver. The difference between the recovered EM field on plane $P$ and the true field on plane $P$ can be attributed to the non-uniqueness of the inverse problem. The recovered field on plane $P$, which is much closer to the target and has higher sensitivity to the target, can potentially be used for enhanced interpretation and inversion. This application will be addressed in our future research. Although the recovered field on plane $P$ is slightly different from the true one, it can be used to re-computed the EM field on the surface but in much denser receiver configuration and the receiver orientation is flexible. Such application will be demonstrated in the following section. 
Previously, we have assumed that there were no nearsurface inhomogeneities in our model and that the EM anomaly was caused by the target only. In practical application of electromagnetic survey, the measured field could be significantly distorted by the nearsurface inhomogeneities, and this effect should be properly treated for the correct interpretation of the observed electromagnetic field data (Hördt and Scholl, 2004). The nearsurface inhomogeneities can be well delineated using conventional methods such as DC electric surveys (Zhdanov, 2009) . Our proposed redatuming method provides an approach to reducing the nearsurface distortion by downward continuation of the observed field from the surface down to a horizontal plane close to the target.

As an example, we have introduced nearsurface inhomogeneities randomly distributed in the $20 \mathrm{~m}$ thick nearsurface layer as shown in Fig. 7 (panel e). This figure also shows a comparison of the EM fields on the surface with and without the effect of the nearsurface inhomogeneities. For a better comparison, we present the amplitude and phase of the fields. One can clearly see the distortions caused by the nearsurface inhomogeneities. The left panels of Fig. 8 show the field on the horizontal plane at a depth of $500 \mathrm{~m}$ for the model with the nearsurface inhomogeneities, while the middle panels present the field on this plane for the model without the nearsurface distortions. One can clearly see that the field on the underground plane is less distorted by the nearsurface inhomogeneities comparing to the field observed on the earth's surface. The right panels of this figure show the EM fields on the underground plane obtained by redatuming for the model with nearsurface distortion. Remarkably, the field produced by redatuming (the right panels) is less distorted by the nearsurface inhomogeneities and it is very close to the true field (the left panels). This result illustrates the robustness of the method for redatuming through the inhomogeneous medium and a possibility of using this technique for reducing the distortion effects of the near surface inhomogeneities.

\section{Redatuming for Kevin Dome BSEM project}

Kevin Dome is a large underground geological structure in Toole County, Montana. The CSEM survey is designed primary to monitor the $\mathrm{CO}_{2}$ sequestration (Zhdanov et al., 2013). In this area, there is an abundance of $\mathrm{CO}_{2}$ naturally occurring that has been trapped in places for millions of years indicating strong cap rock formations. Also, $\mathrm{CO}_{2}$ can be extracted from the top portion of the dome and piped a relatively short distance down the dome's flank and outside the natural $\mathrm{CO}_{2}$ accumulation to the inject site. Fig. 9 is a vertical section for Kevin Dome project.

A 3D resistivity model for the Kevin Dome is constructed based on the well-logs. This model contains 12 layers with resistivity range between 30 to $150 \mathrm{Ohm}-\mathrm{m}$. The $\mathrm{CO}_{2}$ is injected into the Devonian Duperow formation which is our target layer. This layer extends from $1100 \mathrm{~m}$ to $1140 \mathrm{~m}$ in vertical direction. The resistivity of this layer is $66 \mathrm{Ohm}-\mathrm{m}$ with out $\mathrm{CO}_{2}$ and $100 \mathrm{Ohm}-\mathrm{m}$ with $\mathrm{CO}_{2}$. Fig. 10 shows a $3 \mathrm{D}$ resistivity model that we constructed from well-logging data for Kevin Dome. The dots in panel (a) of Fig. 10 show the location of receivers on the earth's surface. The borehole is drilled at the center. A vertical electric bipole is placed inside of the borehole to excited EM fields. The background resistivity is chosen to be $33 \mathrm{Ohm}-\mathrm{m}$.

Originally, the designed EM receivers are placed on the earth's surface for a dense configuration. The spacing of receivers is $200 \mathrm{~m}$ in $x$ and $y$ dimension. As we mentioned before, this kind of dense receiver

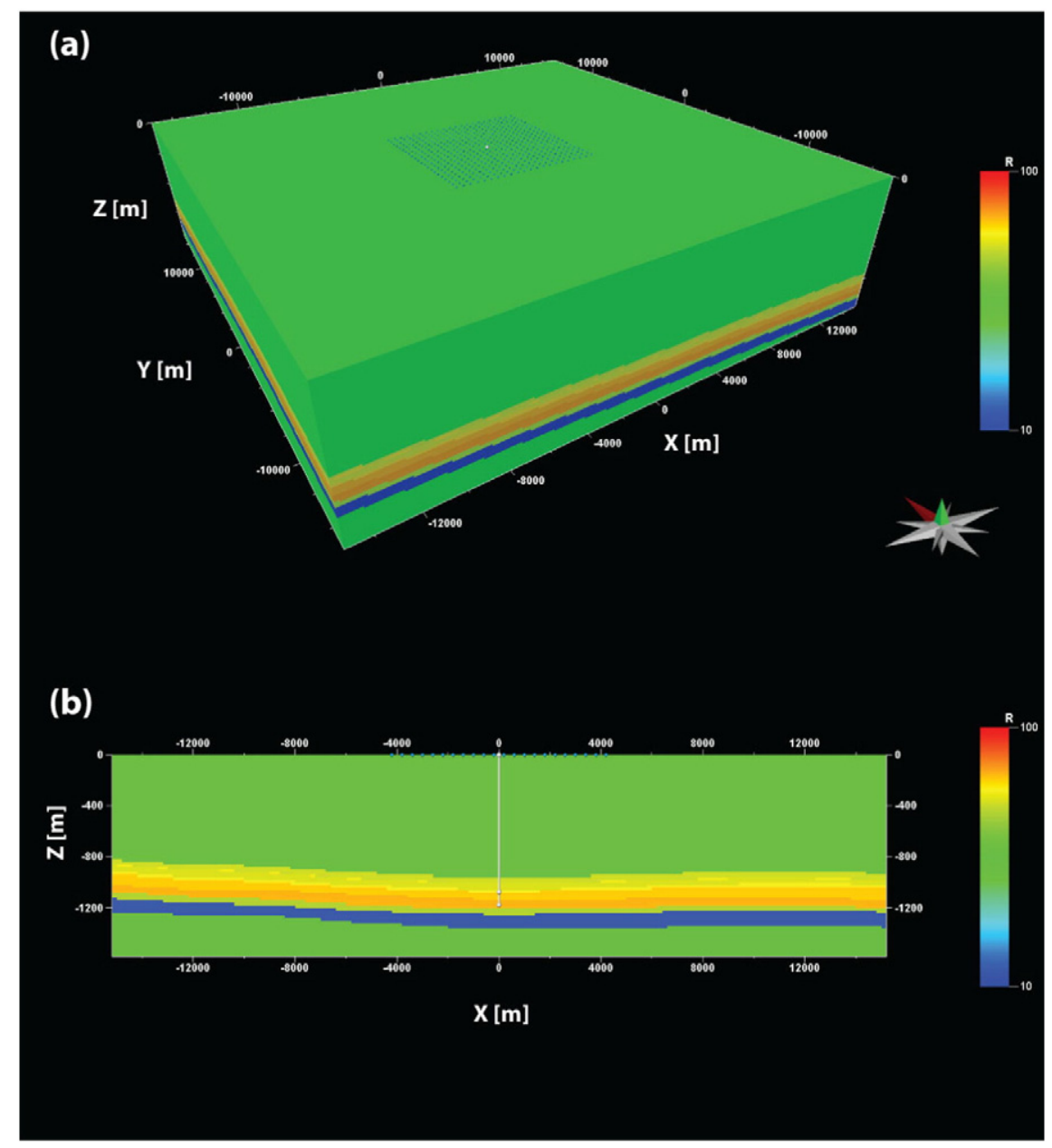

Fig. 10. A 3D resistivity model for Kevin Dome project site. 

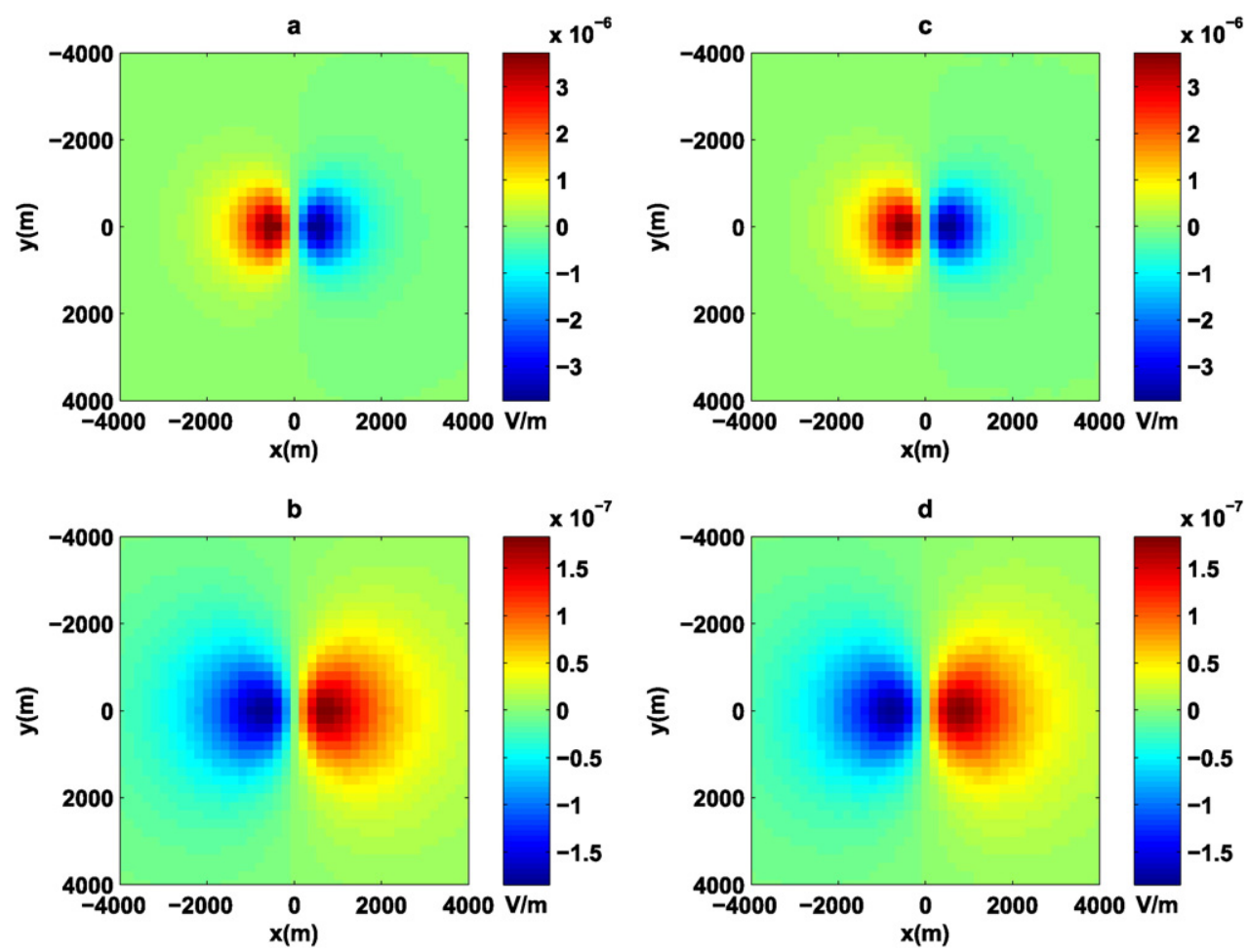

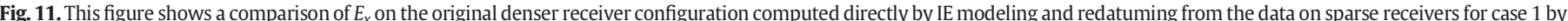

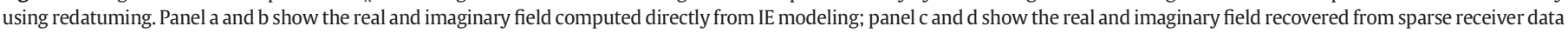
by using redatuming.

configuration is expensive. In this section, we will design the optimized survey configuration based on the redatuming technique. In our approach, we will construct some survey with less receiver and in smaller area to recover the scattering EM field on the original dense receivers. We first find a horizontal plane above the anomalous domain which is large enough. In our numerical study, we choose this plane located
$500 \mathrm{~m}$ under the earth's surface (it also can be chosen at other depth above the target). The EM field on this plane is recovered first by solving the Stratton-Chu type integral equations. In the next step, the EM field on the earth's surface at any location can be computed by applying a linear upward continuation operator (Stratton-Chu integral formula) to the field on the underground horizontal plane. In the following study,
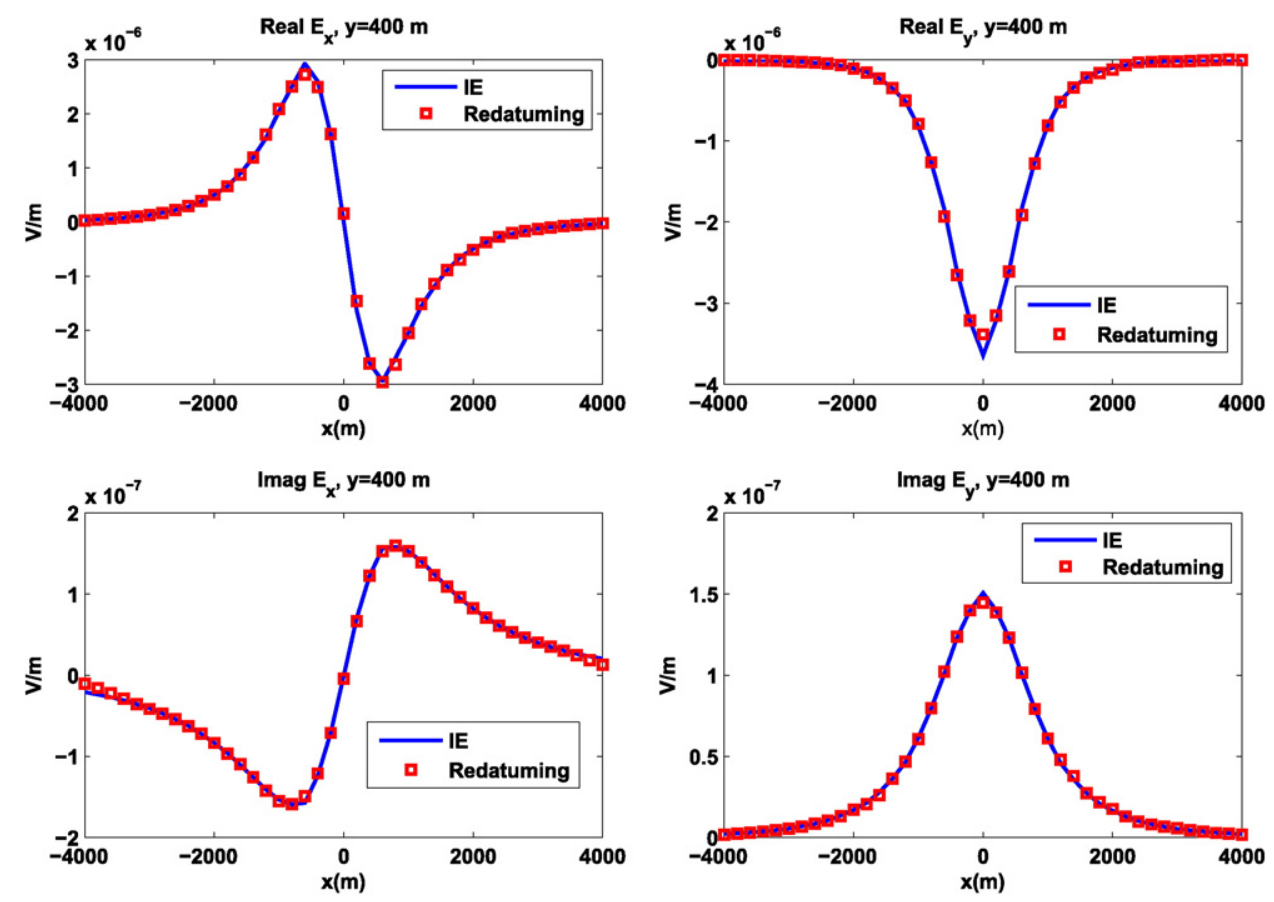

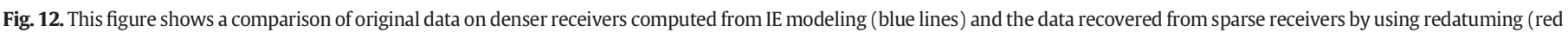
squares) at $y=400 \mathrm{~m}$ for case 2 . 

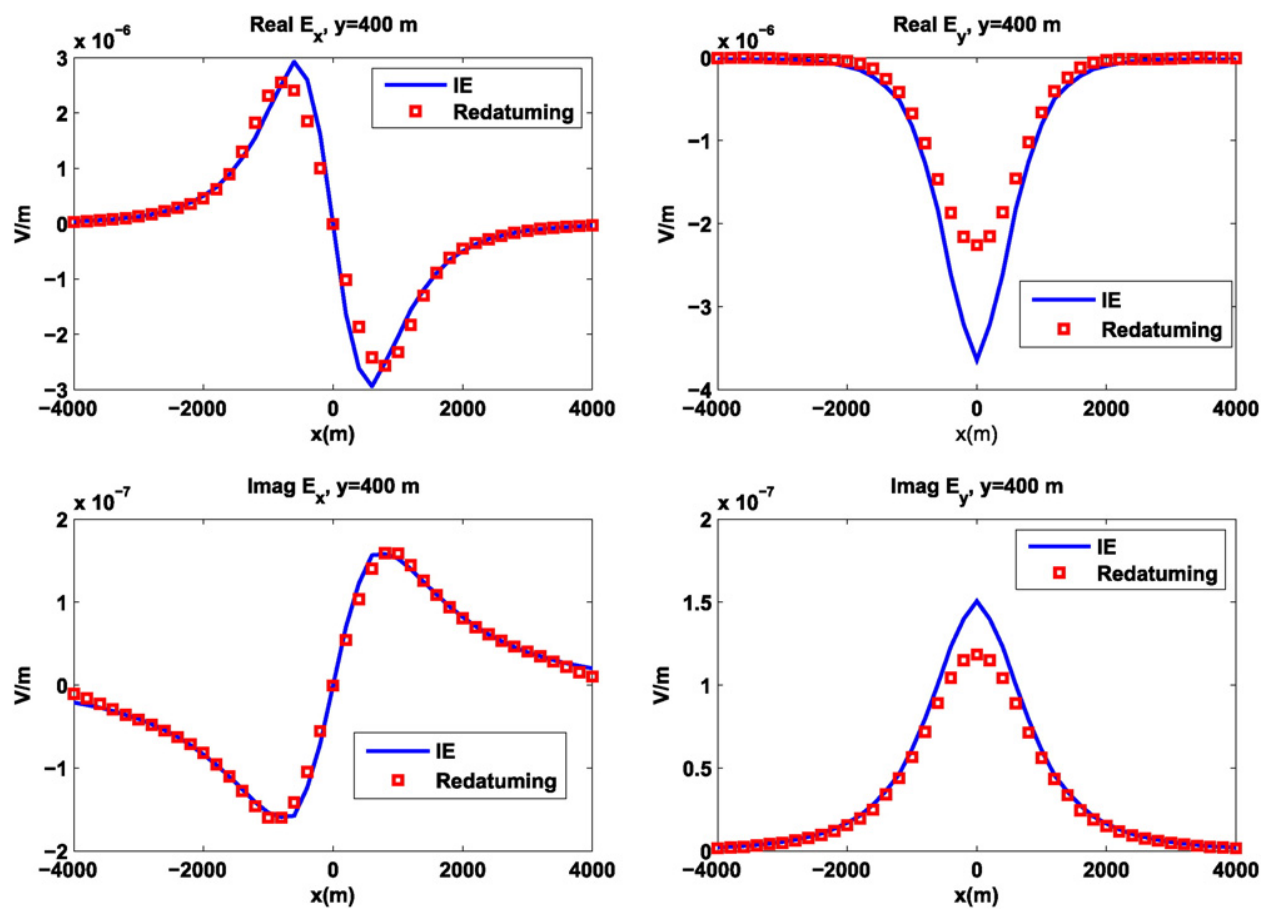

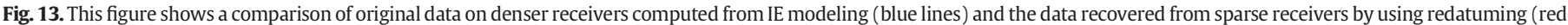
squares) at $y=400 \mathrm{~m}$ for case 3 .

we will generally refer this process as redatuming. Fig. 11, left panels, shows the observed anomalous EM field data on the original dense receivers.

\subsection{Case 1: redatuming from receivers with spacing of $400 \mathrm{~m}$}

In this case, we set the spacing of the EM receivers on the earth's surface to be $400 \mathrm{~m}$ in $x$ and $y$ dimension which is twice as the spacing in the original EM receiver configuration. The EM field on the denser receivers can be recovered from the data spaced at $400 \mathrm{~m}$ in $x$ and $y$ dimension by using redatuming. Fig. 11 shows a comparison of EM field on the earth's surface for the original receiver configuration computed directly by IE modeling and recovered from data on sparse receivers by using redatuming technique. We can see that the field recovered by redatuming method is very close to the true data on the original denser receiver configuration. The study indicates that the original

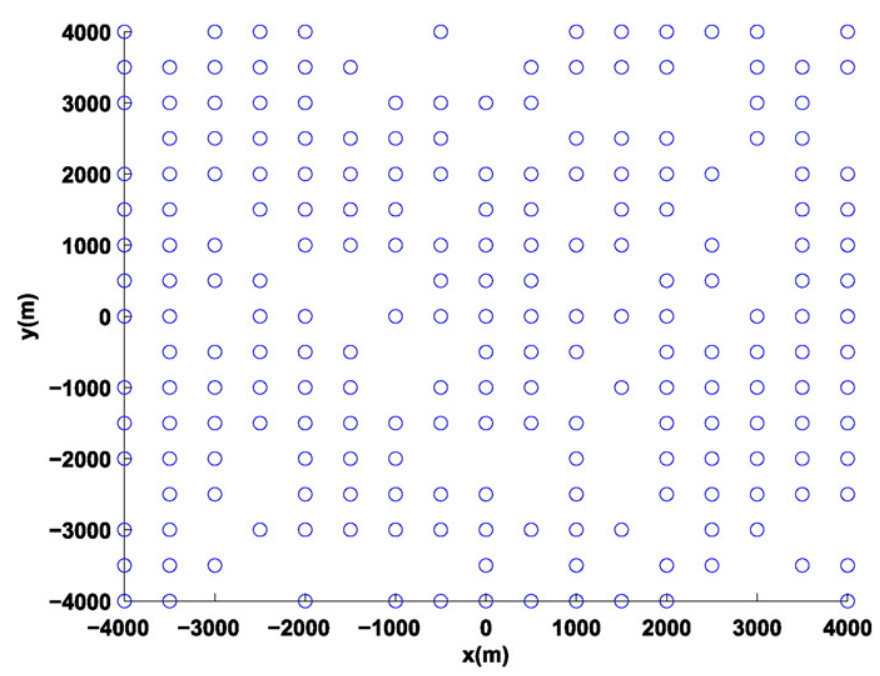

Fig. 14. Irregular grid of the receivers. designed dense receiver configurations with spacing of $200 \mathrm{~m}$ in $x$ and $y$ dimension is unnecessary since it can be well recovered from sparse receiver configuration by using redatuming method.

\subsection{Case 2: redatuming from receivers with spacing of $600 \mathrm{~m}$}

In this case, we set the spacing of the EM receivers on the earth's surface to be $600 \mathrm{~m}$ in $x$ and $y$ dimension which is three times as the spacing in the original EM receiver configuration. Also, the area covered by receivers extends from $-3200 \mathrm{~m}$ to $3200 \mathrm{~m}$ in both $x$ and $y$ dimension which is smaller than the area covered by the original receivers. Fig. 12 shows, as an example, the profiles of recovered data on denser receivers from sparse receivers with $600 \mathrm{~m}$ spacing by using redatuming method compared with the true data from IE modeling for the original dense receiver configuration. We can see that the EM data recovered from sparse receivers based on our redatuming method practically coincide with the true data on denser receivers. Although the sparse receiver configuration covers a smaller area than the original denser receivers, the EM field can be effectively extrapolated by using redatuming method. We can see that the sparse receiver spaced by $600 \mathrm{~m}$ and extended from $-3200 \mathrm{~m}$ to $3200 \mathrm{~m}$ in both $x$ and $y$ dimension will be sufficient to recover the EM data on the originally designed dense EM receiver configuration.

\subsection{Case 3: redatuming from receivers with spacing of $800 \mathrm{~m}$}

In this case, we set the spacing of the EM receivers on the earth's surface to be $800 \mathrm{~m}$ in $x$ and $y$ dimension which is four times as the spacing in the original EM receiver configuration. Also, the area covered by receivers extends from $-3200 \mathrm{~m}$ to $3200 \mathrm{~m}$ in both $x$ and $y$ dimension which is smaller than the area covered by the original receivers. Fig. 13 shows, as an example, the profiles of recovered data on denser receivers from sparse receivers with $800 \mathrm{~m}$ spacing by using redatuming method compared with the true data from IE modeling for the original dense receiver configuration. For this case, it seems that we start to loose accuracy for the interpolation and extrapolation since the available receivers is much less than the original receiver configuration. As such, we conclude 

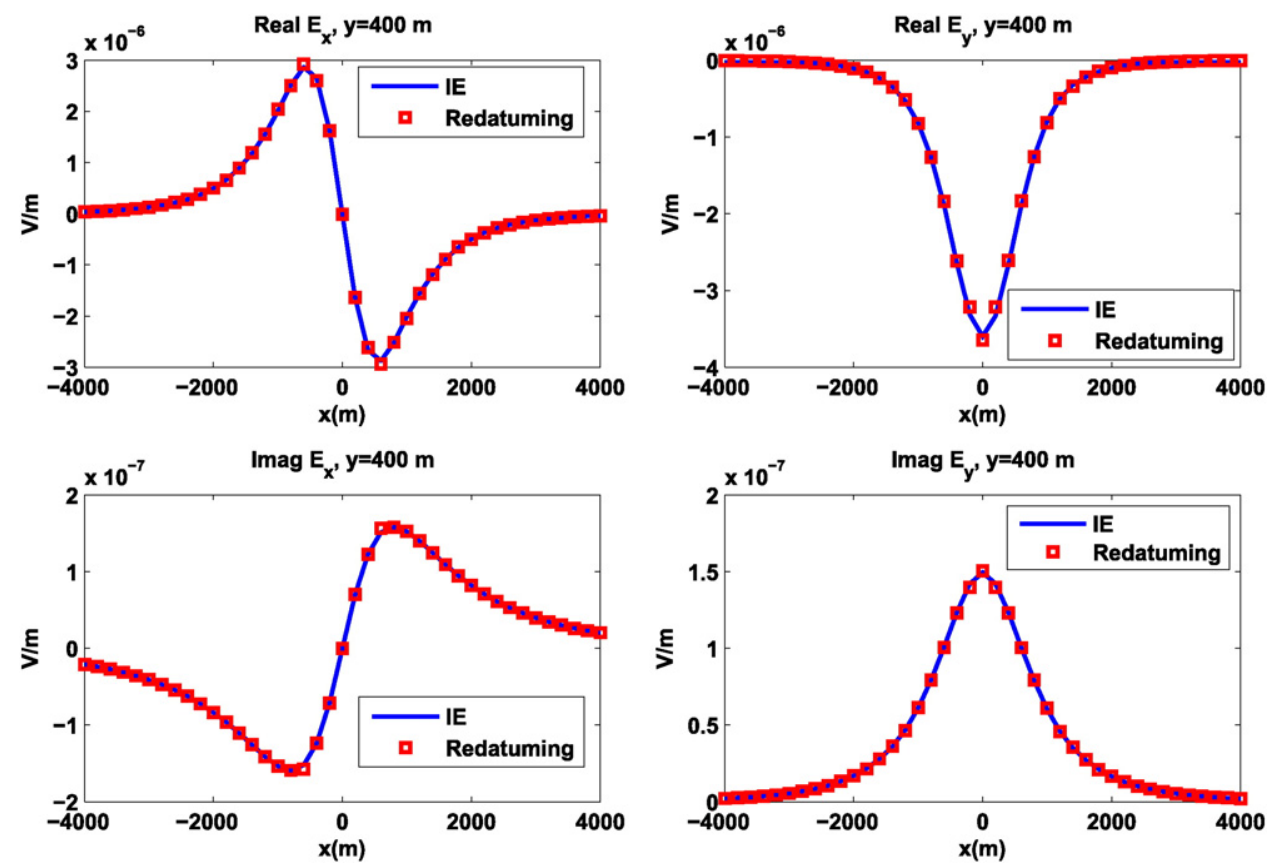

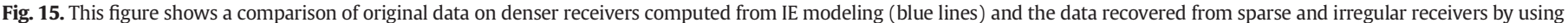
redatuming (red squares) at $y=400 \mathrm{~m}$ for case 4 .

that this survey configuration is too sparse and the receiver configuration in case 2 spaced by $600 \mathrm{~m}$ is the optimized one for the proposed CSEM survey in Kevin Dome EM Project Site.

\subsection{Case 4: redatuming from the field observed on irregular distributed receivers}

Previously, we have assumed that the receivers on the earth's surface are located on a regular grid. However, in a real case, the receivers can be deployed on irregular grids only due to various reasons (availability of difficult-to-access areas, costs optimization, etc.). It is well known that, using the data observed on a regular grid has a significant advantage over analyzing the data on an irregular grid in order to produce a robust inversion result (e.g., Cai and Zhdanov, 2015). In this section, we consider that the data are collected on an irregular grid as shown in Fig. 14 We have applied the redatuming method to recover the EM field in the receivers distributed densely on a regular grid. Fig. 15 shows the profiles of recovered data in the densely distributed receivers in comparison with the true data generated by rigorous IE modeling on the same regular grid. One can clearly see that the recovered EM data on the regular grid are very close to the true data on the regular grid even though the actual receivers were located on a sparse and irregular grid. Thus, we can conclude that redatuming provides a model based method of interpolation of the EM field data.

\section{Conclusions}

We have developed a method of redatuming observed EM data from actual receivers, located on the earth's surface into virtual receivers located at depth. The method is based on using Stratton-Chu type integral transforms. The redatuming is achieved by using the regularized conjugate gradient method of solving an ill-posed inverse problem. The application of the regularization theory makes it possible to apply this method to the noisy observed data. We should also emphasize in the conclusion that the developed redatuming method can be used for the cases with the inhomogeneous background conductivity distribution.

We consider an application of this method for processing BSEM data. By placing virtual receivers close to the top of an $\mathrm{HC}$ reservoir, we generate the synthetic EM data which can be potentially used for locating the reservoir. One of the advantages of the redatuming method is that the number of virtual receivers can be much bigger than the number of the actual receivers on the earth's surface. Once the EM field is found underground by using redatuming, we can use it to re-compute the EM field on the surface but in a much denser receiver configuration. Such approach can be viewed as an model-based interpolation method for electromagnetic field. The method can be used to design the optimized survey configuration as we demonstrated in the study of Kevin Dome EM Project Site.

\section{Acknowledgements}

The authors acknowledge the University of Utah Consortium for Electromagnetic Modeling and Inversion (CEMI), and Technolmaging for the support of this research and permission to publish.

\section{Appendix A. Generalized Lorentz lemma}

This appendix reviews the basic theorem characterizing the relationships between the surface and volume distributions of the EM fields in arbitrary inhomogeneous media. Let us consider two models of EM parameters distribution, Model A with complex conductivity $\widetilde{\sigma}_{A}$ and magnetic permeability $\mu_{A}$, and Model B with complex conductivity $\widetilde{\sigma}_{B}$ and magnetic permeability $\mu_{B}$, respectively. We assume that the frequency-domain EM field $\left\{\mathbf{E}^{A}, \mathbf{H}^{A}\right\}$ in Model $A$ is excited by the electric and magnetic sources, $\left\{\mathbf{j}_{A}^{e}, \mathbf{j}_{A}^{m}\right\}$; while the EM field $\left\{\mathbf{E}^{B}, \mathbf{H}^{B}\right\}$ in Model B is excited by the sources, $\left\{\mathbf{j}_{B}^{e}, \mathbf{j}_{B}^{m}\right\}$, and that both electromagnetic fields have the same frequency $\omega$. These fields satisfy the corresponding Maxwell's equations:

$\nabla \times \mathbf{H}^{A}=\widetilde{\sigma}_{A} \mathbf{E}^{A}+\mathbf{j}_{A}^{e}$

$\nabla \times \mathbf{E}^{A}=i \omega \mu_{A} \mathbf{H}^{A}-\mathbf{j}_{A}^{m}$,

$\nabla \times \mathbf{H}^{B}=\widetilde{\sigma}_{B} \mathbf{E}^{B}+\mathbf{j}_{B}^{e}$ 
$\nabla \times \mathbf{E}^{B}=i \omega \mu_{B} \mathbf{H}^{B}-\mathbf{j}_{B}^{m}$.

Calculating the dot products of Eq. (32) with $\mathbf{E}^{B}$, Eq. (33) with $\mathbf{H}^{B}$, Eq. (34) with $\mathbf{E}^{A}$, and Eq. (35) with $\mathbf{H}^{A}$ we obtain the expression

$$
\begin{aligned}
\nabla \cdot & \left\{\left[\mathbf{E}^{B} \times \mathbf{H}^{A}\right]-\left[\mathbf{E}^{A} \times \mathbf{H}^{B}\right]\right\}=\left(\widetilde{\sigma}_{B}-\widetilde{\sigma}_{A}\right) \mathbf{E}^{A} \cdot \mathbf{E}^{B} \\
& -i \omega\left(\mu_{B}-\mu_{A}\right) \mathbf{H}^{A} \cdot \mathbf{H}^{B}+\mathbf{E}^{A} \cdot \mathbf{j}_{B}^{e}+\mathbf{H}^{B} \cdot \mathbf{j}_{A}^{m}-\mathbf{E}^{B} \cdot \mathbf{j}_{A}^{e}-\mathbf{H}^{A} \cdot \mathbf{j}_{B}^{m} .
\end{aligned}
$$

We consider arbitrary domain $V$, bounded by a closed surface $S$. Integrating (36) over $V$ and applying the Gauss theorem, we find

$$
\begin{aligned}
\iint_{S}\{ & {\left.\left[\mathbf{E}^{B} \times \mathbf{H}^{A}\right]-\left[\mathbf{E}^{A} \times \mathbf{H}^{B}\right]\right\} \cdot d \mathbf{s} } \\
= & \iiint_{V}\left[i \omega \Delta \mu \mathbf{H}^{A} \cdot \mathbf{H}^{B}-\Delta \widetilde{\sigma} \mathbf{E}^{A} \cdot \mathbf{E}^{B}\right] \mathrm{dv} \\
& +\iiint_{V}\left[\mathbf{E}^{A} \cdot \mathbf{j}_{B}^{e}+\mathbf{H}^{B} \cdot \mathbf{j}_{A}^{m}-\mathbf{E}^{B} \cdot \mathbf{j}_{A}^{e}-\mathbf{H}^{A} \cdot \mathbf{j}_{B}^{m}\right] \mathrm{dv},
\end{aligned}
$$

where

$\Delta \widetilde{\sigma}=\widetilde{\sigma}_{A}-\widetilde{\sigma}_{B}, \Delta \mu=\mu_{A}-\mu_{B}$

Eq. (37) represents a mathematical formulation of the generalized Lorentz lemma.

\section{References}

Bakulin, A., Calvert, R., 2006. The virtual source method: theory and case study. Geophysics $71,139-150$.

Berdichevsky, M.N., Zhdanov, M.S., 1984. Advanced Theory of Deep Geomagnetic Sounding. Elsevier.
Cai, H., Zhdanov, M.S., 2015. Application of Cauchy-type integrals in developing effective methods for depth-to-basement inversion of gravity and gravity gradiometry data. Geophysics 80, G81-G94.

Constable, S., 2010. Ten years of marine CSEM for hydrocarbon exploration. Geophysics 75 (75A67-75A81).

He, Z., Hu, W., Dong, W., 2010. Petroleum electromagnetic prospecting advances and case studies in China. Surv. Geophys. 31, 207-224.

He, Z., Liu, X., Qiu, W., Zhou, H., 2005. Mapping reservoir boundary by borehole surface TFEM: two case studies. Lead. Edge 24, 896-900.

Hördt, A., Scholl, C., 2004. The effect of local distortions on timedomain electromagnetic measurements. Geophysics 69, 87-96.

Hunziker, J., Slob, E., Wapenaar, K., 2009. Controlled Source Electromagnetic Interferometry by multidimen-sional deconvolution: spatial sampling aspects in Sea Bed Logging. 71st EAGE Conference and Exhibition, Expanded Abstracts.

Marsala, A.F, Al-Buali, M.H. Ali, Z.A. Ma, S.M., He, Z, Biyan, T, Zhao, G, He, T, 2011a. First pilot of borehole to surface electromagnetic in Saudi Arabia: a new technology to enhance reservoir mapping and monitoring. 73rd EAGE Conference and Exhibition, Expanded Abstracts, pp. 23-26.

Marsala, A.F., Al-Buali, M.H., Ali, Z.A., Ma, S.M., He, Z., Biyan, T., Zhao, G., He, T., 2011b. First borehole to surface electromagnetic survey in KSA: reservoir mapping and monitoring at a new scale. SPE Extended Abstract Presented at the Annual Technical Conference and Exhibition of the Society of Petroleum Engineers, 30 October-2 November 2011.

Schuster, G.T., 2009. Seismic Interferometry. Cambridge University Press.

Schuster, G.T., Zhou, M., 2006. A theoretical overview of model-based and correlationbased redatuming methods. Geophysics 71, 103-110.

Stratton, J.A., 1941. Electromagnetic Theory. McGraw-Hill Book Company.

Wapenaar, K., Draganov, D., Snieder, R., Campman, X., Verdel, A., 2010. Tutorial on seismic interferometry: part 1 - basic principles and applications. Geophysics 75A, 195-209.

Wapenaar, K., Slob, E., Snieder, R., 2008. Seismic and electromagnetic controlled-source interferometry in dissipative media. Geophys. Prospect. 56, 419-434.

Zhdanov, M.S., 1988. Integral Transforms in Geophysics. Springer-Verlag.

Zhdanov, M.S., 2002. Geophysical Inverse Theory and Regularization Problems. Elsevier.

Zhdanov, M.S., 2009. Geophysical Electromagnetic Theory and Methods. Elsevier.

Zhdanov, M.S., 2010. Electromagnetic geophysics: notes from the past and the road ahead. Geophysics 75, A49-A66.

Zhdanov, M.S., Endo, M., Black, N., Spangler, L., Fairweather, S., Hibbs, A., Eiskamp, G.A., Will, R., 2013. Electromagnetic monitoring of CO2 sequestration in deep reservoirs. Firs Break 31, 85-92. 